\title{
RATIONALITY OF BLOCKS OF QUASI-SIMPLE FINITE GROUPS
}

\author{
NIAMH FARRELL AND RADHA KESSAR
}

\begin{abstract}
Let $\ell$ be a prime number. We show that the Morita Frobenius number of an $\ell$-block of a quasi-simple finite group is at most 4 and that the strong Frobenius number is at most $4|D|^{2}$ !, where $D$ denotes a defect group of the block. We deduce that a basic algebra of any block of the group algebra of a quasi-simple finite group over an algebraically closed field of characteristic $\ell$ is defined over a field with $\ell^{a}$ elements for some $a \leq 4$. We derive consequences for Donovan's conjecture. In particular, we show that Donovan's conjecture holds for $\ell$-blocks of special linear groups.
\end{abstract}

\section{INTRODUCTION AND MAIN RESULTS}

Let $\ell$ be a prime number, and let $(K, \mathcal{O}, k)$ be an $\ell$-modular system with $k \cong \overline{\mathbb{F}}_{\ell}$ such that $\mathcal{O}$ is absolutely unramified. The following is the main result of this paper (see Section 2 for notation and definitions).

Theorem 1.1. Let $G$ be a quasi-simple finite group, and let $B$ be a block algebra of $\mathcal{O} G$. Let $D$ be a defect group of $B$.

(i) The Morita Frobenius numbers of $B$ and of $k \otimes_{\mathcal{O}} B$ are at most 4.

(ii) The strong Frobenius number of $B$ is at most $4|D|^{2}$ !.

In many cases in the above theorem we show that the Morita Frobenius number of $B$ is in fact equal to 1 (see Theorem 8.1), and in no case do we show that the number is greater than 1 . We note that there are examples of blocks of $\ell$-solvable groups with Morita Frobenius number equal to 2 [2]. In [19], the first author calculated the Morita Frobenius numbers of $k \otimes_{\mathcal{O}} B$ for several families of blocks $B$ of quasi-simple finite groups. The main outstanding case, which we treat in the current paper, was that of nonunipotent blocks of quasi-simple finite groups of Lie type in nondescribing characteristic.

The motivation for Theorem 1.1 comes from Donovan's conjecture whose statement we recall.

Conjecture 1.2 (Donovan's Conjecture [1, Conjecture M]). Let D be a finite $\ell$ group. There are finitely many Morita equivalence classes of blocks of finite group algebras over $k$ with defect groups isomorphic to $D$.

Received by the editors July 4, 2018, and, in revised form, May 24, 2019.

2010 Mathematics Subject Classification. Primary 20C20; Secondary 20C33.

This article was partly written while the authors were visiting the Mathematical Sciences Research Institute in Berkeley, California in Spring 2018 for the programme Group Representation Theory and Applications supported by the National Science Foundation under Grant No. DMS1440140.

The first author also gratefully acknowledges financial support from the DFG project SFB-TRR 195. 
A weaker version of Donovan's conjecture states that the entries of the Cartan matrices of blocks with defect groups isomorphic to $D$ are bounded by some function which depends only on $|D|$. The gap between the weak and strong forms is precisely a rationality question. This was first observed by Hiss [26] and the theme was developed in [28, Theorem 1.4].

Conjecture 1.3 (Rationality Conjecture [28, Conjecture 1.3]). The Morita Frobenius numbers of blocks of finite group algebras over $k$ with defect groups isomorphic to $D$ are bounded by a function which depends only on $|D|$.

All three conjectures are open at present. The first part of Theorem 1.1 shows that the rationality conjecture holds for blocks of quasi-simple finite groups.

In [27. Theorem 8.6] it was shown that blocks of finite special linear groups satisfy the weak Donovan conjecture. By combining this result with the first part of Theorem 1.1 we obtain Donovan's conjecture for blocks of finite special linear groups. This result first appeared in [20, Theorem C].

Theorem 1.4. Let $D$ be a finite $\ell$-group. There are finitely many Morita equivalence classes of blocks of group algebras over $k$ of finite special linear groups with defect groups isomorphic to $D$.

Motivation for the second part of Theorem 1.1 comes from a recent reduction of Donovan's conjecture for blocks with abelian defect, proved by Eaton and Livesey [16].

Theorem 1.5 ([16, Theorem 1.4]). Suppose that there exist functions $\epsilon, \gamma: \mathbb{N} \rightarrow \mathbb{N}$ such that for all block algebras $B$ of group algebras over $\mathcal{O}$ of quasi-simple finite groups with abelian defect groups of order $\ell^{d}$, the strong Frobenius number of $B$ is bounded by $\epsilon(d)$ and all Cartan invariants of $k \otimes_{\mathcal{O}} B$ are at most $\gamma(d)$. Then Donovan's conjecture holds for blocks with abelian defect groups.

The second part of Theorem 1.1 in combination with the above reduction result shows that in order to prove Donovan's conjecture for blocks with abelian defect groups, it remains to show that the weak Donovan conjecture holds for blocks of quasi-simple finite groups with abelian defect groups. Note that unlike Theorem 1.5. the hypothesis of [16, Theorem 1.4] does not stipulate that $\mathcal{O}$ is absolutely unramified. We also note that Eaton, Eisele, and Livesey recently strengthened the conclusion of Theorem 1.5 to apply to the version of Donovan's conjecture dealing with Morita equivalence classes of blocks of finite group algebras over $\mathcal{O}$ [14].

Recall that a finite-dimensional $k$-algebra $A$ is said to be defined over a subfield $F$ of $k$ if there exists an $F$-algebra $A_{0}$ such that $A \cong k \otimes_{F} A_{0}$. The first part of Theorem 1.1 may be recast as the following rationality statement.

Theorem 1.6. Let $A$ be a basic algebra of a block algebra of $k G$ where $G$ is a quasi-simple finite group. Then $A$ is defined over $\mathbb{F}_{\ell^{a}}$ for some $a \leq 4$.

We begin in Section 2 by recalling some preliminaries including background results on rationality and covering and dominating blocks. Section 3 studies the effect of $\ell$-adic field automorphisms on characters of finite reductive groups in characteristic different from $\ell$. In Section 4 we refine the results of the previous section to the setting of the key reduction theorem of Bonnafé-Dat-Rouquier [5, Section 7]. Theorems 4.4 and 4.5 are then used to prove almost all of the results for blocks of 
the finite groups of Lie type in nondefining characteristic in Section 5 the remaining cases in type $E_{8}$ are dealt with on an ad hoc basis in Section 5.1. Sections 6 and 7 cover the case of defining characteristic, Ree and Suzuki groups, alternating and sporadic groups, and exceptional covering groups. The proofs of Theorem 1.1, Theorem 1.4 and Theorem 1.6 are given in Section 8

\section{Preliminaries}

2.1. Twists through ring automorphisms. Let $R$ be a commutative ring with identity, and let $\varphi: R \rightarrow R$ be a ring automorphism. For an $R$-module $V$, the $\varphi$-twist $V^{\varphi}$ of $V$ is the $R$-module which equals $V$ as a group and where scalar multiplication is given by $\lambda \cdot v=\varphi^{-1}(\lambda) v$, for $\lambda \in R, v \in V$. Denote by $R$-Mod the category of $R$-modules and by $\varphi: R$-Mod $\rightarrow R$-Mod the functor which sends an object $V$ to $V^{\varphi}$ and is the identity on morphisms. Then $\varphi$ is an additive equivalence.

For $A$ an $R$-algebra, we denote by $A^{\varphi}$ the $R$-algebra which is equal to $A^{\varphi}$ as an $R$-module and to $A$ as a ring. The functor $\varphi: R$-Mod $\rightarrow R$-Mod extends to an additive equivalence $\varphi: A$-Mod $\rightarrow A$-Mod. However, the $R$-algebras $A$ and $A^{\varphi}$ are not necessarily Morita equivalent. This gives rise to several invariants, all of which can be thought of as a measure of the rationality of $A$ with respect to $\varphi$.

Definition 2.1. Let $R$ be a commutative unital ring, and let $\varphi: R \rightarrow R$ be an automorphism. Let $A$ be an $R$-algebra.

- The Morita Frobenius number $m f(A)$ of $A$ with respect to $\varphi$ is the least positive integer $m$ (possibly infinity) such that $A^{\varphi^{m}}$ and $A$ are Morita equivalent as $R$-algebras.

- The Frobenius number $f(A)$ of $A$ with respect to $\varphi$ is the least positive integer $m$ (possibly infinity) such that $A^{\varphi^{m}}$ and $A$ are isomorphic as $R$ algebras.

- Let $R \subseteq R^{\prime}$ be an inclusion of unital commutative rings, and let $\varphi^{\prime}: R^{\prime} \rightarrow$ $R^{\prime}$ be a ring automorphism extending $\varphi$. The strong Frobenius number $s f(A)$ of $A$ with respect to $\varphi$ and $\varphi^{\prime}$ is the least positive integer $m$ (possibly infinity) such that there is an $R$-linear isomorphism $\tau: A \rightarrow A^{\varphi^{m}}$ such that the equivalence of module categories induced by $\tau^{\prime}: R^{\prime} \otimes_{R} A \rightarrow R^{\prime} \otimes_{R} A^{\varphi^{m}}$, the unique $R^{\prime}$-linear extension of $\tau$, sends any simple $R^{\prime} \otimes_{R} A$-module $V$ to $V^{\varphi^{\prime m}}$.

It is immediate from the definitions that for any $\varphi$ and $\varphi^{\prime}, m f(A) \leq f(A) \leq$ $s f(A)$. The use of "Frobenius" in the terminology goes back to [2, where Morita Frobenius and Frobenius numbers were defined for finite-dimensional algebras over fields of positive characteristic with $\varphi$ the standard Frobenius isomorphism (see the following subsections). The concept of strong Frobenius numbers is due to Eaton and Livesey [17]; the related concept of $\varphi$-equivalence appeared in [11.

For finite group algebras and their direct factors, $\varphi$-twists have another convenient interpretation. For $\varphi$ and $R$ as above we denote by $\varphi: R G \rightarrow R G$ the ring automorphism which sends an element $\sum_{g \in G} \alpha_{g} g$ of $R G$ to $\sum_{g \in G} \varphi\left(\alpha_{g}\right) g$. Then $\varphi^{-1}: R G \rightarrow(R G)^{\varphi}$ is an isomorphism of $R$-algebras. Further, for any central idempotent $b$ of $R G, \varphi(b)$ is a central idempotent of $R G$ and $\varphi^{-1}$ restricts to an $R$-linear isomorphism $R G \varphi(b) \cong(R G b)^{\varphi}$.

For any function $\chi: G \rightarrow R$ we denote by ${ }^{\varphi} \chi: G \rightarrow R$ the function defined by $\varphi \chi(g)=\varphi(\chi(g)), g \in G$. 
2.2. $\ell$-modular systems and Frobenius maps. Throughout this paper $\ell$ denotes a prime number. Let $(K, \mathcal{O}, k)$ be an $\ell$-modular system with $k \cong \overline{\mathbb{F}}_{\ell}$ such that $\mathcal{O}$ is absolutely unramified, i.e., $J(\mathcal{O})=\ell \mathcal{O}$ (see [39, Chapters 1, 2] for generalities on complete discrete valuation rings).

Let $\sigma: k \rightarrow k$ be the Frobenius automorphism defined by $\lambda \mapsto \lambda^{\ell}, \lambda \in k$. Recall that by the structure theory of complete discrete valuation rings there is a unique automorphism

$$
\hat{\sigma}: \mathcal{O} \rightarrow \mathcal{O}
$$

lifting the Frobenius automorphism $\sigma$ of $k$. The map $\hat{\sigma}$ extends uniquely to an automorphism of $K$. Let $\bar{K}$ be a fixed algebraic closure of $K$. Since all $\ell^{\prime}$-roots of unity in $\bar{K}$ belong to $K$, if $\tau: \bar{K} \rightarrow \bar{K}$ is any automorphism of $\bar{K}$ extending $\hat{\sigma}$, then $\tau(\zeta)=\zeta^{\ell}$ for every $\ell^{\prime}$-root of unity in $\bar{K}$. The following fact is well known, we give a proof for the convenience of the reader.

Lemma 2.2. There exists a field automorphism $\tau: \bar{K} \rightarrow \bar{K}$ extending $\hat{\sigma}$ such that $\tau(\zeta)=\zeta$ for every root of unity $\zeta$ in $\bar{K}$ of $\ell$-power order.

Proof. Let $\zeta \in \bar{K}$ be a root of unity of $\ell$-power order. We claim that there is a unique extension $\tau: K[\zeta] \rightarrow K[\zeta]$ of $\hat{\sigma}$ such that $\tau(\zeta)=\zeta$. Indeed, let $\tau$ : $K[\zeta] \rightarrow K[\zeta]$ be any extension of $\hat{\sigma}$ and suppose that $\tau(\zeta)=\zeta^{i}$. Since $\mathcal{O}$ is unramified, $\operatorname{Gal}(K[\zeta] / K) \cong \operatorname{Aut}(\langle\zeta\rangle)$ (see for instance [29, Lemma 3.3]). Thus there exists $\eta \in \operatorname{Gal}(K[\zeta] / K)$ such that $\eta(\zeta)=\zeta^{i}$. Replacing $\tau$ with $\eta^{-1} \tau$ results in an automorphism which extends $\hat{\sigma}$ and sends $\zeta$ to itself. The uniqueness assertion is obvious and the claim follows. Now let $K_{0} \subset \bar{K}$ be the union of all subfields of the form $K[\zeta]$, where $\zeta$ is a root of unity of $\ell$-power order. Then by a standard Zorn's Lemma argument we have that there is an extension $\tau: K_{0} \rightarrow K_{0}$ of $\hat{\sigma}$ such that $\tau(\zeta)=\zeta$ for any root of unity of $\ell$-power order in $K_{0}$ and hence in $\bar{K}$. Now any extension of $\tau$ to $\bar{K}$ has the desired property.

Henceforth, we fix an extension

$$
\hat{\sigma}: \bar{K} \rightarrow \bar{K}
$$

of $\hat{\sigma}$ to $\bar{K}$ such that $\hat{\sigma}(\zeta)=\zeta$ for every root of unity $\zeta$ in $\bar{K}$ of $\ell$-power order.

2.3. Blocks of finite groups and Frobenius numbers. Let $G$ be a finite group. By a block of $\mathcal{O} G$ (respectively, a block of $k G$ ) we mean a primitive idempotent in $Z(\mathcal{O} G)$ (respectively, $Z(k G)$ ). Recall that the canonical quotient map $\mathcal{O} \rightarrow k$ extends to a surjective ring homomorphism

$$
\pi: \mathcal{O} G \rightarrow k G
$$

and $\pi$ induces a bijection between the set of blocks of $\mathcal{O} G$ and the set of blocks of $k G$. Where there is no ambiguity, we will use the term block or $\ell$-block of $G$ to refer to either a block of $\mathcal{O} G$ or of $k G$.

We denote by $\operatorname{Irr}(G)$ the set of irreducible $\bar{K}$-valued characters of $G$, and for $\chi \in \operatorname{Irr}(G)$, let $e_{\chi}$ denote the central primitive idempotent of $\bar{K} G$ corresponding to $\chi$. For $b$ a block of $\mathcal{O} G$, the set of characters belonging to $b$ or $\pi(b)$ is defined to be

$$
\operatorname{Irr}(b)=\operatorname{Irr}(\pi(b))=\left\{\chi \in \operatorname{Irr}(G) \mid b e_{\chi}=e_{\chi}\right\} .
$$

Lemma 2.3 ([19, Lemma 2.2]). Let $G$ be a finite group, and let $b$ be a block of $\mathcal{O} G$. Then $\pi(\hat{\sigma}(b))=\sigma(\pi(b))$ and $\operatorname{Irr}(\hat{\sigma}(b))=\left\{{ }^{\hat{\sigma}} \chi \mid \chi \in \operatorname{Irr}(b)\right\}$. 
We restate the definitions of the previous subsection by making use of the identification of the twist of a block algebra with another block of the same group algebra. The relevant ring automorphisms are $\sigma$ and $\hat{\sigma}$. Note that when we say that two block algebras $\mathcal{O} G b$ and $\mathcal{O} N c$ are Morita equivalent (or isomorphic), we mean as $\mathcal{O}$-algebras and similarly over $k$.

Definition 2.4. Let $b$ be a block of $\mathcal{O} G$ and let $\mathcal{O} G b$, and $k G \pi(b)=k \otimes_{\mathcal{O}} \mathcal{O} G b$ be the corresponding block algebras.

- The Morita Frobenius number of $k G \pi(b), m f(k G \pi(b))$, is the minimal positive integer $m$ such that $k G \pi(b)$ is Morita equivalent to $k G \sigma^{m}(\pi(b))$.

- The Morita Frobenius number of $\mathcal{O} G b, \operatorname{mf}(\mathcal{O} G b)$, is the minimal positive integer $m$ such that $\mathcal{O} G b$ is Morita equivalent to $\mathcal{O} G \hat{\sigma}^{m}(b)$.

- The Frobenius number of $k G \pi(b), f(k G \pi(b))$, is the minimal positive integer $m$ such that $k G \pi(b) \cong k G \sigma^{m}(\pi(b))$.

- The Frobenius number of $\mathcal{O} G b, f(\mathcal{O} G b)$, is the minimal positive integer $m$ such that $\mathcal{O} G b \cong \mathcal{O} G \hat{\sigma}^{m}(b)$.

- The strong Frobenius number of $\mathcal{O} G b, s f(\mathcal{O} G b)$, is the minimal positive integer $m$ such that there exists an $\mathcal{O}$-algebra isomorphism from $\mathcal{O} G b$ to $\mathcal{O} G \hat{\sigma}^{m}(b)$ which, when extended to an isomorphism $\bar{K} G b \rightarrow \bar{K} G \hat{\sigma}^{m}(b)$, induces a bijection on characters given by $\chi \mapsto \hat{\sigma}^{m} \chi$, for all $\chi \in \operatorname{Irr}(b)$.

The first and third definitions above are equivalent to the definitions in the previous subsection with $R=k, \varphi=\sigma$ and $A=k G \pi(b)$. The second and fourth are equivalent to the definitions in the previous subsection with $R=\mathcal{O}, \varphi=\hat{\sigma}$, and $A=\mathcal{O} G b$. The fifth definition corresponds to the definition in the previous subsection with $R=\mathcal{O}, R^{\prime}=\bar{K}, \varphi=\varphi^{\prime}=\hat{\sigma}$, and $A=\mathcal{O} G b$. Note that the isomorphism type of a simple $\bar{K} G$-module is determined by its character and that if $\chi$ is the character of the simple $\bar{K} G$-module $V$, then ${ }^{\hat{\sigma}^{m}} \chi$ is the character of $V^{\hat{\sigma}^{m}}$ when regarded as a $\bar{K} G$-module via pull back through the map $\hat{\sigma}^{m^{-1}}:(\bar{K} G)^{\hat{\sigma}^{m}} \rightarrow$ $\bar{K} G$.

Since $\mathcal{O} G$ has only finitely many blocks, $f(\mathcal{O} G b)$ is finite for any block $b$. The first part of the following proposition shows that the same is true for the other numbers.

Proposition 2.5. Let $G$ and $N$ be finite groups. Let $b$ be a block of $\mathcal{O} G$, and let $c$ be a block of $\mathcal{O} N$.

(i) $m f(k G \pi(b)) \leq m f(\mathcal{O} G b) \leq f(\mathcal{O} G b) \leq s f(\mathcal{O} G b) \leq|D|^{2} ! f(\mathcal{O} G b)$.

(ii) If $\mathcal{O} G b$ and $\mathcal{O} N c$ are Morita equivalent, then $m f(\mathcal{O} G b)=m f(\mathcal{O} N c)$. If $k G \pi(b)$ and $k N \pi(c)$ are Morita equivalent, then $m f(k G \pi(b))=m f(k N \pi(c))$.

(iii) If $\mathcal{O} G b$ and $\mathcal{O} N$ c are Morita equivalent, then $\operatorname{sf}(\mathcal{O} G b)=s f(\mathcal{O} N c)$.

Proof. The first two inequalities in part (i) follow directly from the definitions, and the last two inequalities are [16, Proposition 2.3 (i)]. If $M$ is an $\mathcal{O} G b \otimes_{\mathcal{O}}(\mathcal{O} N c)^{o p_{-}}$ module, then $M^{\hat{\sigma}^{m}}$ is an $(\mathcal{O} G b)^{\hat{\sigma}^{m}} \otimes_{\mathcal{O}}\left((\mathcal{O} N c)^{o p}\right)^{\hat{\sigma}^{m}} \cong\left(\mathcal{O} G b \otimes_{\mathcal{O}}(\mathcal{O} N c)^{o p}\right)^{\hat{\sigma}^{m}}$ module and $M$ induces a Morita equivalence between $\mathcal{O} G b$ and $\mathcal{O} N c$ if and only if $M^{\hat{\sigma}^{m}}$ induces a Morita equivalence between $(\mathcal{O} G b)^{\hat{\sigma}^{m}}$ and $(\mathcal{O} N c)^{\hat{\sigma}^{m}}$. This proves part (ii) over $\mathcal{O}$. The proof over $k$ is identical. Part (iii) is [16, Proposition 2.3 (ii)].

We will make repeated use of the following result of Linckelmann [32, Theorem 1 and Proposition 2]. 
Proposition 2.6. Let $G$ be a finite group, and let $b$ be a block of $\mathcal{O} G$. If the defect groups of $\mathcal{O} G b$ are cyclic, or if $\ell=2$ and the defect groups of $\mathcal{O} G b$ are Klein four groups, then $\operatorname{sf}(\mathcal{O} G b)=1$.

\subsection{Morita Frobenius numbers and basic algebras of blocks of $k G$.}

Lemma 2.7 ([28, Lemma 2.1]). Let $A$ be a finite-dimensional $k$-algebra. Then $A$ is defined over $\mathbb{F}_{\ell^{m}}$ if and only if $A \cong A^{\sigma^{m}}$.

Two finite-dimensional $k$-algebras are Morita equivalent if and only if their basic algebras are isomorphic (see [31, Section 4.9] for generalities on basic algebras). If $A_{0}$ is a basic algebra of a finite-dimensional $k$-algebra $A$, then $A_{0}^{\sigma^{m}}$ is a basic algebra of $A^{\sigma^{m}}$. We thus obtain the following.

Lemma 2.8. Let $A$ be a finite-dimensional $k$-algebra. The Morita Frobenius number of $A$ is the least positive integer $m$ such that the basic algebras of $A$ are defined over $\mathbb{F}_{\ell^{m}}$.

2.5. Covering and dominating blocks. For $\chi \in \operatorname{Irr}(G)$ we let $b(\chi)$ denote the block of $\mathcal{O} G$ containing $\chi$. If $N \triangleleft G$, then for any $\theta \in \operatorname{Irr}(N)$ and any $\chi \in \operatorname{Irr}(G)$ we use the following notation for the set of irreducible characters of $G$ covering $\theta$, and the set of irreducible characters of $N$ covered by $\chi$, respectively:

$$
\begin{aligned}
& \operatorname{Irr}(G \mid \theta)=\left\{\psi \in \operatorname{Irr}(G): \theta \text { is an irreducible constituent of } \psi_{N}\right\}, \\
& \operatorname{Irr}(N \mid \chi)=\left\{\psi \in \operatorname{Irr}(N): \psi \text { is an irreducible constituent of } \chi_{N}\right\} .
\end{aligned}
$$

Lemma 2.9. Suppose $N \triangleleft G$ are finite groups such that $G / N$ is abelian and let $\theta \in \operatorname{Irr}(N)$. Then for any pair of characters $\chi_{1}, \chi_{2} \in \operatorname{Irr}(G \mid \theta)$, there exists a linear character $\eta \in \operatorname{Irr}(G / N)$ such that $\chi_{2}=\chi_{1} \eta$. Moreover, $\mathcal{O} G b\left(\chi_{1}\right) \cong \mathcal{O} G b\left(\chi_{2}\right)$ as $\mathcal{O}$-algebras.

Proof. The first part follows from [13, Lemma 13.21]. To show the second part, let $\eta \in \operatorname{Irr}(G / N)$ such that $\chi_{2}=\chi_{1} \eta$, and let $\eta^{\prime}$ denote the $\ell^{\prime}$-part of $\eta$. Then $\eta^{\prime}$ takes values in $\mathcal{O}^{\times}$, and the map given by

$$
\sum_{g \in G} \alpha_{g} g \mapsto \sum_{g \in G} \alpha_{g} g \eta\left(g^{-1}\right), \quad \sum_{g \in G} \alpha_{g} g \in \mathcal{O} G
$$

is an $\mathcal{O}$-algebra automorphism of $\mathcal{O} G$ which restricts to an isomorphism between $\mathcal{O} G b\left(\chi_{1}\right)$ and $\mathcal{O} G b\left(\chi_{1} \eta^{\prime}\right)$. Since $\eta$ and $\eta^{\prime}$ agree on $\ell^{\prime}$ elements of $G$,

$$
b\left(\chi_{2}\right)=b\left(\chi_{1} \eta\right)=b\left(\chi_{1} \eta^{\prime}\right)
$$

proving the second assertion.

Recall that if $Z$ is a normal subgroup of a finite group $G$, then a block $\bar{b}$ of $\mathcal{O}(G / Z)$ is said to be dominated by a block $b$ of $\mathcal{O} G$ if $\bar{b} \mu(b) \neq 0$, where $\mu$ is the $\mathcal{O}$-algebra surjection $\mathcal{O} G \rightarrow \mathcal{O}(G / Z)$ induced by the canonical surjection $G \rightarrow G / Z$.

Lemma 2.10. Let $Z$ be a normal subgroup of $G$, and let $\mu: \mathcal{O} G \rightarrow \mathcal{O}(G / Z)$ be the $\mathcal{O}$-algebra homomorphism induced by the canonical surjection map from $G$ to $G / Z$. Then for any block $b$ of $\mathcal{O} G$, either $\mu(b)=0$ or $\mu(b)$ is a sum of blocks of $\mathcal{O}(G / Z)$. Moreover, we have the following:

(i) For any block $c$ of $\mathcal{O}(G / Z)$ there is a unique block $b$ of $\mathcal{O} G$ such that $c \mu(b)$ $\neq 0$. 
(ii) If $Z$ is an $\ell^{\prime}$-group and $\mu(b) \neq 0$, then $\mu$ restricts to an isomorphism $\mathcal{O} G b \cong$ $\mathcal{O}(G / Z) \mu(b)$.

(iii) If $Z$ is an $\ell$-group and $Z \leq Z(G)$, then $\mu$ induces a bijection between the set of blocks of $\mathcal{O} G$ and the set of blocks of $\mathcal{O}(G / Z)$.

(iv) The map $\mu$ commutes with the action of $\hat{\sigma}$.

Proof. The first claim and part (i) follow from the fact that $\mu$ is a surjective homomorphism of $\mathcal{O}$-algebras, whence the image of any central idempotent is either zero or a central idempotent. Suppose that $Z$ is an $\ell^{\prime}$-group and that $\mu(b) \neq 0$. Then $\operatorname{Irr}(b)=\operatorname{Irr}(\mu(b))$ where we regard $\operatorname{Irr}(G / Z)$ as a subset of $\operatorname{Irr}(G)$ via inflation 36. Chapter 5, Theorem 8.8]. Thus, $\mathcal{O} G b$ and $\mathcal{O}(G / Z) \mu(b)$ have the same $\mathcal{O}$-rank and the restriction of $\mu$ to $\mathcal{O} G b$ is injective. Since this restriction is also surjective, we obtain (ii). For a proof of (iii), see [36, Chapter 5, Theorem 8.11]. Part (iv) is immediate from the definitions.

Lemma 2.11. Suppose $N \triangleleft G$ are finite groups such that $G / N \cong C_{2} \times C_{2}$. Then for any $G$-stable linear character $\tau \in \operatorname{Irr}(N)$, its square $\tau^{2}$ extends to a linear character of $G$.

Proof. Let $H \triangleleft G$ be such that $G / H \cong C_{2}$ and $H / N \cong C_{2}$. Since $H / N$ is cyclic and $\tau$ is $G$-stable, and therefore $H$-stable, $\tau$ extends to a linear character $\hat{\tau}$ of $H$.

Suppose that $g \in G$. Then ${ }^{g} \tau=\tau$, so both $\hat{\tau}$ and ${ }^{g} \hat{\tau}$ are elements of $\operatorname{Irr}(H \mid \tau)=$ $\operatorname{Irr}\left(H \mid{ }^{g} \tau\right)$. Thus by Lemma 2.9, there exists a linear character $\lambda \in \operatorname{Irr}(H / N)$ such that ${ }^{g} \hat{\tau}=\lambda \hat{\tau}$. Since $H / N \cong C_{2}, \lambda^{2}=1$.

As $\tau$ is a linear character, $\hat{\tau}^{2}$ is an extension of $\tau^{2}$ to $H$. Therefore ${ }^{g}\left(\hat{\tau}^{2}\right)=$ $\left.{ }^{g} \hat{\tau}\right)^{2}=(\lambda \hat{\tau})^{2}=\hat{\tau}^{2}$. It follows that $\hat{\tau}^{2}$ is $G$-stable, and therefore $\hat{\tau}^{2}$ extends to $G$. Hence, $\tau^{2}$ extends to $G$.

The following easy lemma will be used in the next section.

Lemma 2.12. Let $G$ be a finite group, and let $H$ be a subgroup of $G$. If $\theta: H \rightarrow \bar{K}^{\times}$ is a linear character of $\ell^{\prime}$-order, then ${ }^{\hat{\sigma}} \theta=\theta^{\ell}$.

Proof. Since $\theta$ and ${ }^{\hat{\sigma}} \theta$ have the same kernel, we may assume that $H$ is an abelian $\ell^{\prime}$-group. Then for any $x \in H$, we have

$$
{ }^{\hat{\sigma}} \theta(x)=\sigma(\theta(x))=\theta(x)^{\ell}=\theta^{\ell}(x) .
$$

The following descent result is a consequence of Rickard's theorem on lifting splendid Rickard equivalences from characteristic $\ell$ to characteristic 0 .

Lemma 2.13. Let $\left(K^{\prime}, \mathcal{O}^{\prime}, k^{\prime}\right)$ be an $\ell$-modular system, and let $\mathcal{O}_{0}:=W\left(k^{\prime}\right) \leq \mathcal{O}^{\prime}$ be the Witt vectors of $k^{\prime}$ in $\mathcal{O}^{\prime}$. Let $b$ a central idempotent of $\mathcal{O}_{0} G$, let $H$ be a finite group, and let $c$ be a central idempotent of $\mathcal{O}_{0} H$. Suppose that $C$ is a splendid Rickard complex of $\left(\mathcal{O}^{\prime} G b, \mathcal{O}^{\prime} H c\right)$-bimodules. Then there exists a splendid Rickard complex $C_{0}$ of $\left(\mathcal{O}_{0} G b, \mathcal{O}_{0} H c\right)$-bimodules such that the following holds:

(i) $C \cong \mathcal{O}^{\prime} \otimes_{\mathcal{O}_{0}} C_{0}$ as a complex of $\left(\mathcal{O}^{\prime} G b, \mathcal{O}^{\prime} H c\right)$-bimodules.

(ii) For all $i, H^{i}(C) \cong \mathcal{O}^{\prime} \otimes_{\mathcal{O}_{0}} H^{i}\left(C_{0}\right)$ as $\left(\mathcal{O}^{\prime} G b, \mathcal{O}^{\prime} H c\right)$-bimodules.

If, in addition, $H^{d}(C)$ induces a Morita equivalence between $\mathcal{O}^{\prime} H c$ and $\mathcal{O}^{\prime} G b$ for some $d$, then $H^{i}\left(C_{0}\right)$ induces a Morita equivalence between $\mathcal{O}_{0} H c$ and $\mathcal{O}_{0} G b$ for all $i$. 
Proof. Since $\mathcal{O}_{0}$ and $\mathcal{O}$ have the same residue field, the existence of $C_{0}$ and statement (i) are immediate from the existence and uniqueness assertions of [37, Theorem 5.2]. More specifically, the complex $\bar{C}:=k^{\prime}{\otimes \mathcal{O}^{\prime}} C_{0}$ is a splendid Rickard complex of $(k G \bar{b}, k H \bar{c})$-bimodules where $\bar{b}$ and $\bar{c}$ denote the images of $b$ and $c$ in $k G$ and $k H$, respectively. By [37, Theorem 5.2], there exists a splendid Rickard complex $C_{0}$ of $\left(\mathcal{O}_{0} G b, \mathcal{O}_{0} H c\right)$-bimodules such that $k^{\prime} \otimes_{\mathcal{O}_{0}} C_{0} \cong \bar{C}$. Note that Rickard's theorem is stated under the assumption that the fraction field of $\mathcal{O}_{0}$ be a splitting field for $G$ and $H$, but the proof does not use this - the only ingredient is the completeness of $\mathcal{O}_{0}$ and the fact that $\ell$-permutation modules lift (uniquely) from $k$ to $\mathcal{O}_{0}$. By extension of scalars, $\mathcal{O}^{\prime} \otimes_{\mathcal{O}_{0}} C_{0}$ is a splendid Rickard complex of $\left(\mathcal{O}^{\prime} G b, \mathcal{O}^{\prime} H c\right)$-bimodules. Further,

$$
k \otimes_{\mathcal{O}^{\prime}}\left(\mathcal{O}^{\prime} \otimes_{\mathcal{O}_{0}} C_{0}\right) \cong k \otimes_{\mathcal{O}_{0}} C_{0}
$$

as $\left(\mathcal{O}^{\prime} G b, \mathcal{O}^{\prime} H c\right)$-bimodules. Statement (i) now follows by [37, Theorem 5.2], and $C$ is the unique splendid Rickard complex lifting $\bar{C}$.

Statement (ii) follows from (i) since $\mathcal{O}_{0} \subseteq \mathcal{O}^{\prime}$ is a flat extension. The final statement follows from an application of the Noether-Deuring theorem (see for instance [29, Prop. 4.5]).

\section{Galois aCtions And Lusztig SERIES}

We continue with the notation of the previous section. There is a canonical embedding (as valuation fields) of $\mathbb{Q}_{\ell}$ in $K$. Let $\overline{\mathbb{Q}}_{\ell}$ denote the algebraic closure of $\mathbb{Q}_{\ell}$ in $\bar{K}$. We fix a prime $p$ different from $\ell$, a group isomorphism $\iota:(\mathbb{Q} / \mathbb{Z})_{p^{\prime}} \rightarrow \overline{\mathbb{F}}_{p}^{\times}$, and an injective group homomorphism $\jmath:(\mathbb{Q} / \mathbb{Z}) \rightarrow \overline{\mathbb{Q}}_{\ell}^{\times}$.

3.1. Characters of tori and duality. Let $\mathbf{G}$ be a connected reductive algebraic group defined over $\overline{\mathbb{F}}_{p}$ with Frobenius endomorphism $F: \mathbf{G} \rightarrow \mathbf{G}$ (see Remark 4.6). We fix an $F$-stable maximal torus $\mathbf{T}_{0}$ of $\mathbf{G}$, a triple $\left(\mathbf{G}^{*}, \mathbf{T}_{0}^{*}, F\right)$ dual to the triple $\left(\mathbf{G}, \mathbf{T}_{0}, F\right)$, and an $F$-equivariant isomorphism $X\left(\mathbf{T}_{0}\right) \cong Y\left(\mathbf{T}_{0}^{*}\right)$ which sends simple roots to simple coroots as in [13, Definition 13.10], where $X\left(\mathbf{T}_{0}\right)$ denotes the set of characters of $\mathbf{T}_{0}$ and $Y\left(\mathbf{T}_{0}^{*}\right)$ denotes the set of cocharacters of $\mathbf{T}_{0}^{*}$. We let $\nabla(\mathbf{G}, F)$ denote the set of pairs $(\mathbf{T}, \theta)$ where $\mathbf{T}$ is an $F$-stable maximal torus of $\mathbf{G}$ and $\theta: \mathbf{T}^{F} \rightarrow \overline{\mathbb{Q}}_{\ell}$ is a linear character. Dually, let $\nabla^{*}\left(\mathbf{G}^{*}, F\right)$ denote the set of pairs $\left(\mathbf{T}^{*}, s\right)$ where $\mathbf{T}^{*}$ is an $F$-stable maximal torus of $\mathbf{G}^{*}$ and $s \in \mathbf{T}^{* F}$.

The choice of $\iota, \jmath$ and the isomorphism $X\left(\mathbf{T}_{0}\right) \cong Y\left(\mathbf{T}_{0}^{*}\right)$ above determine a bijection [13, 11.15, Proposition 13.13]

$$
\nabla(\mathbf{G}, F) / \mathbf{G}^{F} \rightarrow \nabla^{*}\left(\mathbf{G}^{*}, F\right) / \mathbf{G}^{* F} .
$$

If $(\mathbf{T}, \theta) \in \nabla(\mathbf{G}, F)$ and $\left(\mathbf{T}^{*}, s\right) \in \nabla^{*}\left(\mathbf{G}^{*}, F\right)$, then we write $(\mathbf{T}, \theta) \stackrel{\mathrm{G}}{\leftrightarrow}\left(\mathbf{T}^{*}, s\right)$ if the classes of $(\mathbf{T}, \theta)$ and $\left(\mathbf{T}^{*}, s\right)$ correspond under the above bijection. We write $\mathbf{T} \stackrel{\mathrm{G}}{\leftrightarrow} \mathbf{T}^{*}$ if $\mathbf{T}$ is an $F$-stable maximal torus of $\mathbf{G}$ and $\mathbf{T}^{*}$ is an $F$-stable maximal torus of $\mathbf{G}^{*}$ such that $(\mathbf{T}, \theta) \stackrel{\mathbf{G}}{\leftrightarrow}\left(\mathbf{T}^{*}, s\right)$ for some $\theta$ and some $s$. For $s$ a semisimple element of $\mathbf{G}^{* F}$ we denote by $\nabla(\mathbf{G}, F, s)$ the set of all pairs $(\mathbf{T}, \theta)$ such that $(\mathbf{T}, \theta) \stackrel{\mathbf{G}}{\leftrightarrow}\left(\mathbf{T}^{*}, s\right)$ for some $F$-stable maximal torus $\mathbf{T}^{*}$ of $\mathbf{G}^{*}$ containing $s$. 
The following lemma lists some well-known properties of bijection (1). We give a short indication of the proof for the convenience of the reader. Let

$$
\tau: \mathbf{G}_{s c} \rightarrow[\mathbf{G}, \mathbf{G}]
$$

be a simply connected covering (see [10, Section 8.1]).

Lemma 3.1. Let $s$ be a semisimple element of $\mathbf{G}^{* F}$.

(i) If $(\mathbf{T}, \theta) \in \nabla(\mathbf{G}, F, s)$, then for any $n \in \mathbb{N},\left(\mathbf{T}, \theta^{n}\right) \in \nabla\left(\mathbf{G}, F, s^{n}\right)$.

(ii) There exists an isomorphism $Z\left(\mathbf{G}^{*}\right)^{F} \rightarrow \operatorname{Irr}\left(\mathbf{G}^{F} / \tau\left(\mathbf{G}_{s c}^{F}\right)\right),(t \mapsto \hat{t})$ such that for all $t \in Z\left(\mathbf{G}^{*}\right)^{F}, \nabla(\mathbf{G}, F, t)=\left\{\left(\mathbf{T}, \hat{t}_{\mathbf{T}^{F}}\right)\right\}$, where $\mathbf{T}$ runs over all $F$-stable maximal tori of $\mathbf{G}$.

Proof. Let $\mathbf{T}$ be an $F$-stable maximal torus of $\mathbf{G}$. By construction of the bijection (11), if $\mathbf{T}^{*}$ is an $F$-stable maximal torus of $\mathbf{G}^{*}$ such that $\mathbf{T} \stackrel{\mathrm{G}}{\leftrightarrow} \mathbf{T}^{*}$, then there exists an isomorphism $\zeta: \mathbf{T}^{* F} \rightarrow \operatorname{Irr}\left(\mathbf{T}^{F}\right)$ such that $(\mathbf{T}, \zeta(t)) \stackrel{\mathrm{G}}{\leftrightarrow}\left(\mathbf{T}^{*}, t\right)$ for all $t \in \mathbf{T}^{* F}$ (see [10, (8.15)]). Now suppose that $(\mathbf{T}, \theta) \stackrel{\mathbf{G}}{\leftrightarrow}\left(\mathbf{T}^{*}, s\right)$. Then $(\mathbf{T}, \theta)$ is $\mathbf{G}^{F}$-conjugate to $(\mathbf{T}, \zeta(s))$, hence $\left(\mathbf{T}, \theta^{n}\right)$ is $\mathbf{G}^{F}$-conjugate to $\left(\mathbf{T}, \zeta\left(s^{n}\right)\right)$ and $\left(\mathbf{T}, \zeta\left(s^{n}\right)\right) \stackrel{\mathrm{G}}{\leftrightarrow}\left(\mathbf{T}^{*}, s^{n}\right)$, proving (i).

By [10, (8.19)], there exists an isomorphism $Z\left(\mathbf{G}^{*}\right)^{F} \rightarrow \operatorname{Irr}\left(\mathbf{G}^{F} / \tau\left(\mathbf{G}_{s c}^{F}\right)\right),(t \mapsto \hat{t})$ such that for any $\mathbf{T}, \mathbf{T}^{*}$, and $\zeta$ as above, and any $t \in Z\left(\mathbf{G}^{*}\right)^{F} \leq \mathbf{T}^{* F}$, we have that $\hat{t}_{\mathbf{T}^{F}}=\zeta(t)$, where we view $\hat{t}$ as a character of $\mathbf{G}^{F}$ via pull back through $\mathbf{G}^{F} \rightarrow$ $\mathbf{G}^{F} / \tau\left(\mathbf{G}_{s c}^{F}\right)$ (see [12, Proposition 5.11(ii)] for the independence of the isomorphism). It follows that for any $t \in Z\left(\mathbf{G}^{*}\right)^{F},\left(\mathbf{T}, \hat{t}_{\mathbf{T}^{F}}\right)=(\mathbf{T}, \zeta(t)) \stackrel{\leftrightarrow}{\leftrightarrow}\left(\mathbf{T}^{*}, t\right)$, and hence $\left(\mathbf{T}, \hat{t}_{\mathbf{T}^{F}}\right) \in \nabla(\mathbf{G}, F, t)$.

Now suppose that $(\mathbf{T}, \theta) \in \nabla(\mathbf{G}, F, t)$, say $(\mathbf{T}, \theta) \stackrel{\mathbf{G}}{\leftrightarrow}\left(\mathbf{T}^{*}, t\right)$. Then $(\mathbf{T}, \theta)$ is $\mathbf{G}^{F}$-conjugate to $(\mathbf{T}, \zeta(t))=\left(\mathbf{T}, \hat{t}_{\mathbf{T}^{F}}\right)$. But $\hat{t}$ is a linear character of $\mathbf{G}^{F}$, so it is $\mathbf{G}^{F}$-stable, hence $(\mathbf{T}, \theta)=\left(\mathbf{T}, \hat{t}_{\mathbf{T}^{F}}\right)$, proving part (ii).

Definition 3.2. Let $H$ be a finite group, and let $h \in H$ be an $\ell^{\prime}$-element. We denote by $a_{H}(h)$ the order of $h$ modulo $Z(H)$, and by $r_{H}(h)$ the multiplicative order of $\ell$ modulo $a_{H}(h)$.

Lemma 3.3. Let $s \in \mathbf{G}^{* F}$ be a semisimple $\ell^{\prime}$-element, and let $r=r_{\mathbf{G}^{* F}}(s)$.

(i) Let $(\mathbf{T}, \theta) \in \nabla(\mathbf{G}, F)$. Then $(\mathbf{T}, \theta) \in \nabla(\mathbf{G}, F, s)$ if and only if $\left(\mathbf{T},{ }^{\hat{\sigma}} \theta\right) \in$ $\nabla\left(\mathbf{G}, F, s^{\ell}\right)$

(ii) Let $\tau$ denote the linear character of $\mathbf{G}^{F}$ corresponding to $s^{\ell^{r}-1}$ by the isomorphism in Lemma 3.11(ii). Then ${ }^{\hat{\sigma}^{r}} \theta=\theta \tau_{\mathbf{T}^{F}}$ for all $(\mathbf{T}, \theta) \in \nabla(\mathbf{G}, F, s)$.

Proof. Part (i) follows from Lemma 2.12 and Lemma 3.1(i). To prove (ii), note that by definition of $r, s^{\ell^{r}-1} \in Z\left(\mathbf{G}^{* F}\right)=Z\left(\mathbf{G}^{*}\right)^{F}$, hence $\tau$ is well-defined. Let $(\mathbf{T}, \theta) \in \nabla(\mathbf{G}, F, s)$. By Lemma 3.1(i), $\left(\mathbf{T}, \theta^{\ell^{r}-1}\right) \in\left(\mathbf{G}, F, s^{\ell^{r}-1}\right)$. Hence, by Lemma 3.1 (ii), $\theta^{\ell^{r}-1}=\theta \tau_{\mathbf{T}^{F}}$. Thus

$$
\hat{\sigma}^{r} \theta=\theta^{\ell^{r}}=\theta \theta^{\ell^{r}-1}=\theta \tau_{\mathbf{T}^{F}},
$$

where the first equality holds by Lemma 2.12 .

3.2. Regular embeddings. We fix a regular embedding $i: \mathbf{G} \rightarrow \widetilde{\mathbf{G}}$ (see [10, Section 15.1]) and let $F$ denote a Steinberg morphism on $\widetilde{\mathbf{G}}$ compatible with the Steinberg morphism $F$ on $\mathbf{G}$. The map $\mathbf{T} \mapsto Z(\widetilde{\mathbf{G}}) \mathbf{T}$ induces a bijection between the set of ( $F$-stable) maximal tori of $\mathbf{G}$ and the set of ( $F$-stable) maximal tori 
of $\widetilde{\mathbf{G}}$. The inverse bijection is given by $\widetilde{\mathbf{T}} \mapsto \widetilde{\mathbf{T}} \cap \mathbf{G}$. Set $\widetilde{\mathbf{T}}_{0}=Z(\widetilde{\mathbf{G}}) \mathbf{T}_{0}$. Let $\left(\widetilde{\mathbf{G}}^{*}, \widetilde{\mathbf{T}}_{0}^{*}, F\right)$ be dual to $\left(\widetilde{\mathbf{G}}, \widetilde{\mathbf{T}}_{0}, F\right)$, and let $i^{*}: \widetilde{\mathbf{G}}^{*} \rightarrow \mathbf{G}^{*}$ be a surjection dual to $i$ with $i^{*}\left(\widetilde{\mathbf{T}}_{0}^{*}\right)=\mathbf{T}_{0}^{*}$. Fix an isomorphism $X\left(\widetilde{\mathbf{T}}_{0}\right) \rightarrow Y\left(\widetilde{\mathbf{T}}_{0}^{*}\right)$ lifting the isomorphism $X\left(\mathbf{T}_{0}\right) \rightarrow Y\left(\mathbf{T}_{0}^{*}\right)$.

Lemma 3.4. Let $\tilde{s}$ be a semisimple element of $\widetilde{\mathbf{G}}^{* F}$, and let $s=i^{*}(\tilde{s})$. Let $\widetilde{\mathbf{T}}$ be an F-stable maximal torus of $\widetilde{\mathbf{G}}$, let $\tilde{\theta}: \operatorname{Irr}\left(\widetilde{\mathbf{T}}^{F}\right) \rightarrow \overline{\mathbb{Q}}_{\ell}$ be a linear character of $\widetilde{\mathbf{T}}^{F}$, and let $\theta$ denote the restriction of $\tilde{\theta}$ to $\mathbf{T}^{F}$. If $(\widetilde{\mathbf{T}}, \tilde{\theta}) \in \nabla(\widetilde{\mathbf{G}}, F, \tilde{s})$, then $(\mathbf{T}, \theta) \in \nabla(\mathbf{G}, F, s)$.

Proof. See [4, Lemme 9.3 (a)].

We also record for future use the following fact.

Lemma 3.5. Let $\tilde{s} \in \widetilde{\mathbf{G}}^{* F}$ be a semisimple element and set $s=i^{*}(\tilde{s})$. Then $a_{\widetilde{\mathbf{G}}^{* F}}(\tilde{s})=a_{\mathbf{G}^{* F}}(s)$ and $r_{\widetilde{\mathbf{G}}^{* F}}(\tilde{s})=r_{\mathbf{G}^{* F}}(s)$.

Proof. Let $a=a_{\mathbf{G}^{* F}}(s)$. Then $i^{*}\left(\tilde{s}^{a}\right)=s^{a} \in Z\left(\mathbf{G}^{* F}\right) \leq Z\left(\mathbf{G}^{*}\right)$. Now since $i^{*}: \widetilde{\mathbf{G}}^{*} \rightarrow \mathbf{G}^{*}$ is a surjective homomorphism with kernel a central torus of $\widetilde{\mathbf{G}}^{*}$ (see [4, Proposition 2.5]), $i^{*-1}\left(Z\left(\mathbf{G}^{*}\right)\right)=Z\left(\widetilde{\mathbf{G}}^{*}\right)$. Hence $\tilde{s}^{a} \in Z\left(\widetilde{\mathbf{G}}^{*}\right) \cap \widetilde{\mathbf{G}}^{* F}=Z\left(\widetilde{\mathbf{G}}^{* F}\right)$, showing that $a_{\widetilde{\mathbf{G}}_{* F}}(\tilde{s}) \leq a_{\mathbf{G}^{* F}}(s)$. The reverse inequality is immediate from the fact that the restriction of $i^{*}$ to $\widetilde{\mathbf{G}}^{* F}$ is surjective (see [4, Corollaire 2.7]).

3.3. Lusztig series. For a semisimple element $s$ of $\mathbf{G}^{* F}$, we denote by $\mathcal{E}\left(\mathbf{G}^{F}, s\right) \subseteq$ $\operatorname{Irr}\left(\mathbf{G}^{F}\right)$ the rational Lusztig series corresponding to the $\mathbf{G}^{* F}$-conjugacy class of $s$. Recall that $\mathcal{E}\left(\mathbf{G}^{F}, s\right)$ is the set consisting of the irreducible characters for which $\left\langle\chi, R_{\mathbf{T}}^{\mathbf{G}}(\theta)\right\rangle \neq 0$ for some $(\mathbf{T}, \theta) \in \nabla(\mathbf{G}, F, s)$. By results of Lusztig, the series $\mathcal{E}\left(\mathbf{G}^{F}, s\right)$ as $s$ runs over a set of representatives of $\mathbf{G}^{F}$-conjugacy classes of semisimple elements of $\mathbf{G}^{F}$ partition $\operatorname{Irr}\left(\mathbf{G}^{F}\right)$.

Lemma 3.6. Let $\chi \in \operatorname{Irr}\left(\mathbf{G}^{F}\right)$, let $(\mathbf{T}, \theta) \in \nabla(\mathbf{G}, F)$, and let $s \in \mathbf{G}^{* F}$ be a semisimple $\ell^{\prime}$-element.

(i) For any $\varphi \in \operatorname{Aut}(\bar{K}),\left\langle{ }^{\varphi} \chi, R_{\mathbf{T}}^{\mathbf{G}}\left({ }^{\varphi} \theta\right)\right\rangle=\left\langle\chi, R_{\mathbf{T}}^{\mathbf{G}}(\theta)\right\rangle$.

(ii) $\chi \in \mathcal{E}\left(\mathbf{G}^{F}, s\right)$ if and only if ${ }^{\hat{\sigma}} \chi \in \mathcal{E}\left(\mathbf{G}^{F}, s^{\ell}\right)$.

Proof. By the character formula [13, Proposition 12.2], ${ }^{\varphi} R_{\mathbf{T}}^{\mathbf{G}}(\theta)=R_{\mathbf{T}}^{\mathbf{G}}(\varphi \theta)$. Hence,

$$
\left\langle\chi, R_{\mathbf{T}}^{\mathbf{G}}(\theta)\right\rangle={ }^{\varphi}\left\langle\chi, R_{\mathbf{T}}^{\mathbf{G}}(\theta)\right\rangle=\left\langle{ }^{\varphi} \chi,{ }^{\varphi} R_{\mathbf{T}}^{\mathbf{G}}(\theta)\right\rangle=\left\langle{ }^{\varphi} \chi, R_{\mathbf{T}}^{\mathbf{G}}\left({ }^{\varphi} \theta\right)\right\rangle,
$$

where the second equality holds since $R_{\mathbf{T}}^{\mathbf{G}}(\theta)$ is a generalised character. This proves (i). Part (ii) follows from (i) and Lemma 3.3(i).

The next lemma recalls a consequence of the Jordan decomposition of characters. Recall that for a $\overline{\mathbb{Q}}_{\ell}$-valued class function $\chi$ of $\mathbf{G}^{F}$ the uniform projection of $\chi$ is the orthogonal projection (with respect to $\langle$,$\rangle ) of \chi$ onto the subspace of class functions generated by $R_{\mathbf{T}}^{\mathbf{G}}(\theta),(\mathbf{T}, \theta) \in \nabla(\mathbf{G}, F)$.

Lemma 3.7. Suppose that $Z(\mathbf{G})$ is connected and let $s$ be a semisimple element of $\mathbf{G}^{* F}$. Suppose that $C_{\mathbf{G}^{*}}(s)$ has all classical components. Then each $\chi \in \mathcal{E}\left(\mathbf{G}^{F}, s\right)$ is uniquely determined by its uniform projection.

Proof. The hypothesis implies that the unipotent characters of $C_{\mathbf{G}^{*}}(s)^{F}$ are uniquely determined by their uniform projections 34, 8.1(a)]. Then by Jordan decomposition ([33, 4.23]; see also [10, Theorem 15.8]) each $\chi \in \mathcal{E}\left(\mathbf{G}^{F}, s\right)$ is uniquely determined by its uniform projection. 
Lemma 3.8. Let $s \in \mathbf{G}^{* F}$ be a semisimple element, and let $\varphi \in \operatorname{Aut}(\bar{K})$. Suppose that $C_{\mathbf{G}^{*}}^{\circ}(s)$ has only classical components. Suppose further that there exists $\tilde{s} \in$ $\widetilde{\mathbf{G}}^{* F}$ with $i^{*}(\tilde{s})=s$ and a linear character $\tilde{\eta} \in \operatorname{Irr}\left(\widetilde{\mathbf{G}}{ }^{F}\right)$ such that $\varphi \widetilde{\theta}=\widetilde{\theta} \tilde{\eta}_{\widetilde{\mathbf{T}}^{F}}$ for all $(\widetilde{\mathbf{T}}, \widetilde{\theta}) \in \nabla(\widetilde{\mathbf{G}}, F, \tilde{s})$. Then for any $\chi \in \mathcal{E}\left(\mathbf{G}^{F}, s\right)$, there exists an $x \in \widetilde{\mathbf{G}}^{F}$ such that

$$
{ }^{\varphi} \chi={ }^{x}\left(\chi \tilde{\eta}_{\mathbf{G}^{F}}\right) \text {. }
$$

Proof. Let $\chi \in \mathcal{E}\left(\mathbf{G}^{F}, s\right)$, and let $\tilde{\chi} \in \operatorname{Irr}\left(\widetilde{\mathbf{G}}^{F} \mid \chi\right) \cap \mathcal{E}\left(\widetilde{\mathbf{G}}^{F}, \tilde{s}\right)$ (for the existence of $\tilde{\chi}$ see [4, Proposition 11.7]). Let $(\widetilde{\mathbf{T}}, \tilde{\theta}) \in \nabla(\widetilde{\mathbf{G}}, F, \tilde{s})$. By assumption,

$$
\left\langle\tilde{\chi}, R_{\widetilde{\mathbf{T}}}^{\widetilde{\mathbf{G}}}(\tilde{\theta})\right\rangle=\left\langle\left\langle^{\varphi} \tilde{\chi}, R_{\widetilde{\mathbf{T}}}^{\widetilde{\mathbf{G}}}\left({ }^{\varphi} \tilde{\theta}\right)\right\rangle=\left\langle^{\varphi} \tilde{\chi}, R_{\widetilde{\mathbf{T}}}^{\widetilde{\mathbf{G}}}\left(\tilde{\theta} \tilde{\eta}_{\widetilde{\mathbf{T}}^{F}}\right)\right\rangle,\right.
$$

where the first equality holds by Lemma 3.6. On the other hand, since $\tilde{\eta} \in \operatorname{Irr}\left(\widetilde{\mathbf{G}}^{F}\right)$ is linear,

$$
\left\langle\tilde{\chi}, R_{\widetilde{\mathbf{T}}}^{\widetilde{\mathbf{G}}}(\tilde{\theta})\right\rangle=\left\langle\tilde{\chi} \tilde{\eta}, R_{\widetilde{\mathbf{T}}}^{\widetilde{\mathbf{G}}}(\tilde{\theta}) \tilde{\eta}\right\rangle=\left\langle\tilde{\chi} \tilde{\eta}, R_{\widetilde{\mathbf{T}}}^{\widetilde{\mathbf{G}}}\left(\tilde{\theta} \tilde{\eta}_{\widetilde{\mathbf{T}}^{F}}\right)\right\rangle
$$

where now the second equality holds by the character formula [13, Proposition 12.2]. Since $\left\langle\tilde{\chi}, R_{\widetilde{\mathbf{T}}}^{\widetilde{\mathbf{G}}}(\tilde{\theta})\right\rangle=0$ for all $(\widetilde{\mathbf{T}}, \tilde{\theta}) \in \nabla(\widetilde{\mathbf{G}}, F) \backslash \nabla(\widetilde{\mathbf{G}}, F, \tilde{s})$, it follows that $\varphi \tilde{\chi}$ and $\tilde{\chi} \tilde{\eta}$ have the same uniform projections. By definition $Z\left(\widetilde{\mathbf{G}}^{*}\right)$ is connected, so $C_{\widetilde{\mathbf{G}}^{*}}(\tilde{s})$ is connected. Since $C_{\mathbf{G}^{*}}^{\circ}(s)$ has all classical components the same is true of $C_{\widetilde{\mathbf{G}}^{*}}(\tilde{s})$. Hence by Lemma 3.7. ${ }^{\varphi} \tilde{\chi}=\tilde{\chi} \tilde{\eta}$. It follows that ${ }^{\varphi} \chi$ and $\chi \tilde{\eta}_{\mathbf{G}^{F}}$ are both elements of $\operatorname{Irr}\left(\left.\mathbf{G}^{F}\right|^{\varphi} \tilde{\chi}\right)=\operatorname{Irr}\left(\mathbf{G}^{F} \mid \tilde{\chi} \tilde{\eta}\right)$, proving the result.

3.4. Blocks. For $s \in \mathbf{G}^{* F}$ a semisimple $\ell^{\prime}$-element we denote by $\mathcal{E}_{\ell}\left(\mathbf{G}^{F}, s\right)$ the union of the rational Lusztig series $\mathcal{E}\left(\mathbf{G}^{F}, t\right)$, where $t$ runs over a set of representatives of $\mathbf{G}^{* F}$-conjugacy classes of the semisimple elements of $\mathbf{G}^{* F}$ whose $\ell^{\prime}$-part is $\mathbf{G}^{* F}$-conjugate to $s$. We recall that by results of Broué-Michel and Hiss [10, Theorem 9.12], $\mathcal{E}_{\ell}\left(\mathbf{G}^{F}, s\right)$ is the union of the irreducible characters in some subset of the set of blocks of $\mathcal{O} \mathbf{G}^{F}$, and if $b$ is an $\ell$-block of $\mathcal{O} \mathbf{G}^{F}$ such that $\operatorname{Irr}(b) \subseteq \mathcal{E}_{\ell}\left(\mathbf{G}^{F}, s\right)$, then $\operatorname{Irr}(b) \cap \mathcal{E}\left(\mathbf{G}^{F}, s\right) \neq \emptyset$. If $\operatorname{Irr}(b) \subseteq \mathcal{E}_{\ell}\left(\mathbf{G}^{F}, s\right)$ we say " $b$ is in $\mathcal{E}_{\ell}\left(\mathbf{G}^{F}, s\right)$ " and write $b \in \mathcal{E}_{\ell}\left(\mathbf{G}^{F}, s\right)$.

Since the largest order of semisimple elements of the groups $\mathbf{G}^{* F^{n}}$ become arbitrarily large as $n \rightarrow \infty$, it is easy to see from the above discussion that there is no finite subfield $k_{0}$ of $k$ such that $b \in k_{0} \mathbf{G}^{F^{n}}$ for all $n$ and all blocks $b$ of $k \mathbf{G}^{F^{n}}$. We give an explicit example below.

Example 3.9. Let $\mathbf{G}=\mathrm{PGL}_{r}\left(\overline{\mathbb{F}}_{p}\right), r \geq 2$, and let $F: \mathbf{G} \rightarrow \mathbf{G}$ be the standard Frobenius over $\mathbb{F}_{p}$ with $\mathbf{G}^{F^{n}} \cong \operatorname{PGL}_{r}\left(p^{n}\right)$, and $\mathbf{G}^{*}=\mathrm{SL}_{r}\left(\overline{\mathbb{F}}_{p}\right), \mathbf{G}^{* F^{n}} \cong \mathrm{SL}_{r}\left(p^{n}\right)$ for $n \in \mathbb{N}$. Choose a strictly increasing sequence of positive integers $\left(n_{i}\right)_{i \in \mathbb{N}}$ such that $\left|\mathbf{G}^{* F^{n_{i}}}\right|_{\ell}=\left|\mathbf{G}^{* F^{n_{j}}}\right|_{\ell}$ for all $i, j$ and set $G_{i}=\mathbf{G}^{F^{n_{i}}}, G_{i}^{*}=\mathbf{G}^{* F^{n_{i}}}$. For each $i$, let $\lambda_{i} \in \mathbb{F}_{p^{n_{i}}}$ be an element of order $\left(p^{n_{i}}-1\right)_{\ell^{\prime}}$, and let $s_{i} \in G_{i}^{*}$ be a diagonal matrix with diagonal entries $\lambda_{i}, \lambda_{i}^{-1}, 1, \ldots, 1$. Let $d_{i}$ be the smallest positive integer such that $s_{i}^{\ell^{d_{i}}}$ is $\mathbf{G}^{*}$-conjugate to $s_{i}$. Since $\left(p^{n_{i}}-1\right)_{\ell^{\prime}} \rightarrow \infty$ as $i \rightarrow \infty$, we have that $d_{i} \rightarrow \infty$ as $i \rightarrow \infty$.

For each $i \in \mathbb{N}$, choose a block $b_{i}$ of $k G_{i}$ in $\mathcal{E}_{\ell}\left(G_{i}, s_{i}\right)$ and a character $\chi_{i} \in$ $\mathcal{E}\left(G_{i}, s_{i}\right) \cap \operatorname{Irr}\left(b_{i}\right)$. By Lemma [3.6, ${ }^{\hat{\sigma}^{m}} \chi \in \mathcal{E}\left(G_{i}, s_{i}^{\ell^{m}}\right)$ for $m \in \mathbb{N}$. Hence by Lemma 2.3, $\sigma^{m}\left(b_{i}\right)$ is in $\mathcal{E}_{\ell}\left(G_{i}, s_{i}^{\ell^{m}}\right)$. It follows that if $b_{i}=\sigma^{m}\left(b_{i}\right)$, then $m$ is divisible by $d_{i}$. Hence the smallest subfield $k_{0}$ of $k$ such that $b_{i} \in k_{0} G$ contains at least $p^{d_{i}}$ elements. In particular, there exists no finite subfield $k_{0}$ of $k$ such that $b_{i} \in k_{0} G$ for all $i$. Since the $\ell$-part of the order of the $G_{i}$ 's is equal, this also shows 
that there is no finite subfield $k_{0}$ of $k$ such that $b$ belongs to $k_{0} G$ for all blocks $b$ of $k G$ with a given defect.

\section{Applications of the Bonnafé-Dat-Rouquier theorem}

We keep all the notation of the sections before Example 3.9. In particular, $\mathbf{G}$ is a connected reductive algebraic group defined over $\overline{\mathbb{F}}_{p}$ with Frobenius endomorphism $F: \mathbf{G} \rightarrow \mathbf{G}$. We fix a finite extension $\mathbb{Q}_{\ell} \subseteq K^{\prime} \subseteq \bar{K}$ of $\mathbb{Q}_{\ell}$ in $\bar{K}$ such that $K^{\prime}$ is a splitting field for all sections of $\mathbf{G}^{F}$ and of $\mathbf{G}^{* F}$, and let $\mathcal{O}^{\prime}$ be the ring of integers of $K^{\prime}$ over $\mathbb{Z}_{\ell}$.

The proof of our main theorem relies in a fundamental way on the reduction theorem of [5. Section 7] which we now describe. Let $s \in \mathbf{G}^{* F}$ be a semisimple $\ell^{\prime}$-element. Let $\mathbf{L}^{*}=C_{\mathbf{G}^{*}}\left(Z^{\circ}\left(C_{\mathbf{G}^{*}}^{\circ}(s)\right)\right)$ be the minimal Levi subgroup of $\mathbf{G}^{*}$ containing $C_{\mathbf{G}^{*}}^{\circ}(s)$, and let $\mathbf{L}$ be a Levi subgroup of $\mathbf{G}$ dual to $\mathbf{L}^{*}$ as in [13, Chapter 13]. Since $s \in \mathbf{G}^{* F}, \mathbf{L}^{*}$ and $\mathbf{L}$ are $F$-stable. Further, the duality between $\mathbf{G}$ and $\mathbf{G}^{*}$ induces an $F$-equivariant isomorphism $N_{\mathbf{G}}(\mathbf{L}) / \mathbf{L} \rightarrow N_{\mathbf{G}^{*}}\left(\mathbf{L}^{*}\right) / \mathbf{L}^{*},\left(w \mapsto w^{*}\right)$ and a bijection $\nabla(\mathbf{L}, F) / \mathbf{L}^{F} \rightarrow \nabla^{*}\left(\mathbf{L}^{*}, F\right) / \mathbf{L}^{* F}$ which is equivariant with respect to the action of $\left(N_{\mathbf{G}}(\mathbf{L}) / \mathbf{L}\right)^{F}$ on $\nabla(\mathbf{L}, F) / \mathbf{L}^{F}$ and the action of $\left(N_{\mathbf{G}^{*}}\left(\mathbf{L}^{*}\right) / \mathbf{L}^{*}\right)^{F}$ on $\nabla^{*}\left(\mathbf{L}^{*}, F\right) / \mathbf{L}^{* F}$ : for all $w \in\left(N_{\mathbf{G}}(\mathbf{L}) / \mathbf{L}\right)^{F},(\mathbf{T}, \theta) \in \nabla(\mathbf{L}, F)$, and $\left(\mathbf{T}^{*}, s\right) \in$ $\nabla^{*}\left(\mathbf{L}^{*}, F\right)$,

$$
(\mathbf{T}, \theta) \stackrel{\mathbf{L}}{\mapsto}\left(\mathbf{T}^{*}, s\right) \Longleftrightarrow w(\mathbf{T}, \theta) \stackrel{\mathbf{L}}{\leftrightarrow} w^{*}\left(\mathbf{T}^{*}, s\right) .
$$

Note that we identify $\left(N_{\mathbf{G}}(\mathbf{L}) / \mathbf{L}\right)^{F}$ and $N_{\mathbf{G}^{F}}(\mathbf{L}) / \mathbf{L}^{F}$. Let $N^{*}=C_{\mathbf{G}^{*}}(s)^{F}$...' and define $N$ to be the subgroup of $N_{\mathbf{G}}(\mathbf{L})$ containing $\mathbf{L}$ such that $N / \mathbf{L}$ corresponds to $N^{*} / \mathbf{L}^{*}$ via the above isomorphism between $N_{\mathbf{G}^{*}}\left(\mathbf{L}^{*}\right) / \mathbf{L}^{*}$ and $N_{\mathbf{G}}(\mathbf{L}) / \mathbf{L}$. As discussed in [5, Section 7A], $N^{F} / \mathbf{L}^{F} \cong N^{* F} / \mathbf{L}^{* F} \subseteq C_{\mathbf{G}^{*}}(s)^{F} / C_{\mathbf{G}^{*}}^{\circ}(s)^{F}$. Let $e_{s}^{\mathbf{G}^{F}}$ (respectively, $e_{s}^{\mathbf{L}^{F}}$ ) denote the sum of all blocks of $\mathcal{O}^{\prime} \mathbf{G}^{F}$ (respectively, $\mathcal{O}^{\prime} \mathbf{L}^{F}$ ) in $\mathcal{E}_{\ell}\left(\mathbf{G}^{F}, s\right)$ (respectively, $\mathcal{E}_{\ell}\left(\mathbf{L}^{F}, s\right)$ ).

Let $\mathbf{V}$ be the unipotent radical of a parabolic subgroup of $\mathbf{G}$ containing $\mathbf{L}$ as a Levi subgroup, and let $\mathbf{Y}_{\mathbf{V}}=\left\{g \mathbf{V} \in \mathbf{G} / \mathbf{V} \mid g^{-1} F(g) \in \mathbf{V} . F(\mathbf{V})\right\}$ be the corresponding Deligne-Lusztig $(\mathbf{G}, \mathbf{L})$-variety [5, 2.E]. Let $G \Gamma_{c}\left(Y_{\mathbf{V}}, \mathcal{O}^{\prime}\right)^{\text {red }}$ be the complex of $\ell$-permutation $\left(\mathcal{O}^{\prime} \mathbf{G}^{F}, \mathcal{O}^{\prime} \mathbf{L}^{F}\right)$-bimodules defined in [5, 2.A, 2.C].

Theorem 4.1 ([5, Theorem 7.7]). Suppose that $N^{F} / \mathbf{L}^{F}$ is a cyclic group. The complex $G \Gamma_{c}\left(Y_{\mathbf{V}}, \mathcal{O}^{\prime}\right)^{\text {red }} e_{s}^{\mathbf{L}^{F}}$ extends to a splendid Rickard complex $C$ of $\left(\mathcal{O}^{\prime} \mathbf{G}^{F} e_{s}^{\mathbf{G}^{F}}\right.$, $\mathcal{O}^{\prime} N^{F} e_{s}^{\mathbf{L}^{F}}$ )-bimodules.

There is a bijection $b \mapsto b^{\prime}$ between blocks of $\mathcal{O}^{\prime} \mathbf{G}^{F}$ in $\mathcal{E}_{\ell}\left(\mathbf{G}^{F}\right.$, s) and blocks of $\mathcal{O}^{\prime} N^{F}$ covering blocks of $\mathcal{O}^{\prime} \mathbf{L}^{F}$ in $\mathcal{E}_{\ell}\left(\mathbf{L}^{F}, s\right)$ determined by the rule $b C=C b^{\prime}$. Given a block $b$ of $\mathcal{O}^{\prime} \mathbf{G}^{F}$ in $\mathcal{E}_{\ell}\left(\mathbf{G}^{F}, s\right)$, the complex $b C b^{\prime}$ is a splendid Rickard complex of $\left(\mathcal{O}^{\prime} \mathbf{G}^{F} b, \mathcal{O}^{\prime} N^{F} b^{\prime}\right)$-bimodules and such that $H^{\operatorname{dim}\left(\mathbf{Y}_{\mathbf{V}}\right)}\left(b C b^{\prime}\right)$ induces a Morita equivalence between $\mathcal{O}^{\prime} \mathbf{G}^{F} b$ and $\mathcal{O}^{\prime} N^{F} b^{\prime}$.

The above Morita equivalences descend to unramified discrete valuation rings and are compatible with taking quotients by central $\ell$-subgroups. Let $k^{\prime}$ be the residue field of $\mathcal{O}^{\prime}$, and let $\mathcal{O}_{0}:=W\left(k^{\prime}\right) \subseteq \mathcal{O}^{\prime}$ be the ring of Witt vectors of $k^{\prime}$ in $\mathcal{O}^{\prime}$.

Proposition 4.2. Keep the notation and hypothesis of Theorem 4.1, Let $b$ be a block of $\mathcal{O}^{\prime} \mathbf{G}^{F}$ in $\mathcal{E}_{\ell}\left(\mathbf{G}^{F}, s\right)$, and let $b^{\prime}$ be the block of $\mathcal{O}^{\prime} N^{F}$ in bijection with $b$. 
Let $Z \leq Z\left(\mathbf{G}^{F}\right)$ be an $\ell$-group, and let $\bar{b}$ (respectively, $\left.\bar{b}^{\prime}\right)$ be the block of $\mathcal{O}^{\prime}\left(\mathbf{G}^{F} / Z\right)$ (respectively, $\mathcal{O}^{\prime}\left(N^{F} / Z\right)$ ) dominated by $b$ (respectively, $\left.\bar{b}^{\prime}\right)$. Then $\mathcal{O}_{0}\left(\mathbf{G}^{F} / Z\right) \bar{b}$ and $\mathcal{O}_{0}\left(N^{F} / Z\right) \bar{b}^{\prime}$ are Morita equivalent. Consequently, $\mathcal{O}\left(\mathbf{G}^{F} / Z\right) \bar{b}$ and $\mathcal{O}\left(N^{F} / Z\right) \bar{b}^{\prime}$ are Morita equivalent.

Proof. Note that $Z \leq Z\left(\mathbf{L}^{F}\right)$. By Theorem 4.1 and Lemma 2.13, there exists an $\left(\mathcal{O}_{0} \mathbf{G}^{F} b, \mathcal{O}_{0} N^{F} b^{\prime}\right)$-bimodule $M$ such that $M$ induces a Morita equivalence between $\mathcal{O}_{0} \mathbf{G}^{F} b$ and $\mathcal{O}_{0} N^{F} b^{\prime}$ and such that $\mathcal{O}^{\prime} \otimes \mathcal{O} M \cong H^{\operatorname{dim}\left(\mathbf{Y}_{\mathbf{V}}\right)}\left(b C b^{\prime}\right)$ as $\left(\mathcal{O}^{\prime} \mathbf{G}^{F} b, \mathcal{O}^{\prime} N^{F} b^{\prime}\right)$ bimodules.

For any integer $i, H^{i}\left(b C b^{\prime}\right)$ is isomorphic to a summand of $H^{i}(C)$ and

$$
\operatorname{Res}_{\mathbf{G}^{F} \times \mathbf{L}^{F}}^{\mathbf{G}^{F} \times N^{F}} H^{i}(C)=H^{i}\left(G \Gamma_{c}\left(Y_{\mathbf{V}}, \mathcal{O}^{\prime}\right)^{\operatorname{red}} e_{s}^{\mathbf{L}^{F}}\right)
$$

is isomorphic to a summand of $H^{i}\left(G \Gamma_{c}\left(Y_{\mathbf{V}}, \mathcal{O}^{\prime}\right)^{\mathrm{red}}\right)$. By [5, 2.C]

$$
H^{i}\left(G \Gamma_{c}\left(Y_{\mathbf{V}}, \mathcal{O}^{\prime}\right)^{\text {red }}\right) \cong H^{i}\left(G \Gamma_{c}\left(Y_{\mathbf{V}}, \mathcal{O}^{\prime}\right)\right) \cong H^{i}\left(R \Gamma_{c}\left(Y_{\mathbf{V}}, \mathcal{O}^{\prime}\right)\right) \text {. }
$$

For any $z \in Z$ and any $g \mathbf{V} \in \mathbf{Y}_{\mathbf{V}}$, we have $z . g \mathbf{V}=g \mathbf{V}$.z. Hence, by the functoriality of $\ell$-adic cohomology with respect to finite morphisms, $z u=u z$ for all $u \in H^{i}\left(R \Gamma_{c}\left(Y_{\mathbf{V}}, \mathcal{O}^{\prime}\right)\right)$ and $z \in Z$. By the above discussion it follows that $z u=u z$ for all $u \in M$ and $z \in Z$. Now the first assertion result follows from [15, Lemma 2.7]. The second is immediate from the first as $\mathcal{O}_{0} \subseteq \mathcal{O}$.

Thus the problem of bounding the Morita Frobenius number of $b$ is reduced to the problem of bounding the Morita Frobenius number of $b^{\prime}$. This in turn can be bounded by appealing to the rationality of unipotent character values. The two theorems below give instances of this philosophy in action.

Let $i: \mathbf{G} \hookrightarrow \widetilde{\mathbf{G}}$ be a regular embedding as in Section 3.1 with dual $i^{*}: \widetilde{\mathbf{G}}^{*} \rightarrow \mathbf{G}^{*}$. Let $\widetilde{\mathbf{L}}=Z(\widetilde{\mathbf{G}}) \mathbf{L}$ be a Levi subgroup of $\widetilde{\mathbf{G}}$, and let $\widetilde{\mathbf{L}}^{*}$ be the full inverse image of $\mathbf{L}^{*}$ under $i^{*}$. Then $Z(\widetilde{\mathbf{L}})$ is connected by [4, Corollaire 4.4] and $[\widetilde{\mathbf{L}}, \widetilde{\mathbf{L}}]=[\mathbf{L}, \mathbf{L}]$; hence the restriction of $i$ to $\mathbf{L}$ is a regular embedding of $\mathbf{L}$ into $\widetilde{\mathbf{L}}$ with dual $i^{*}: \widetilde{\mathbf{L}}^{*} \rightarrow \mathbf{L}^{*}$.

Lemma 4.3. The group $\widetilde{\mathbf{L}}^{F}$ normalises $N^{F}$.

Proof. Since $\widetilde{\mathbf{L}}=Z(\widetilde{\mathbf{G}}) \mathbf{L}$ and $N \subseteq N_{\mathbf{G}}(\mathbf{L})$, it is clear that $[\widetilde{\mathbf{L}}, N] \subseteq \mathbf{L}$ and hence $\left[\widetilde{\mathbf{L}}^{F}, N^{F}\right] \subseteq \mathbf{L}^{F} \subseteq N^{F}$

Recall the number $r_{H}(h)$ as introduced in Definition 3.2

Theorem 4.4. Keep the notation and hypothesis of Proposition 4.2. Let $r=$ $r_{\mathbf{L}^{* F}}(s)$ and suppose that $C_{\mathbf{L}^{*}}^{\circ}(s)$ has all classical components. Then

$$
\mathcal{O}\left(N^{F} / Z\right) \hat{\sigma}^{r}\left(\overline{b^{\prime}}\right) \cong \mathcal{O}\left(N^{F} / Z\right){\overline{b^{\prime}}}^{\prime}
$$

Proof. Let $\tilde{s}$ be a semisimple $\ell^{\prime}$-element of $\widetilde{\mathbf{L}}^{* F}$ with $i^{*}(\tilde{s})=s$. By Lemma 3.5 $\tilde{t}:=\tilde{s}^{\ell^{r}-1} \in Z\left(\widetilde{\mathbf{L}}^{*}\right)^{F}$. Let $\tilde{\tau}$ be the linear character of $\widetilde{\mathbf{L}}^{F}$ corresponding to $\tilde{t}$ as in

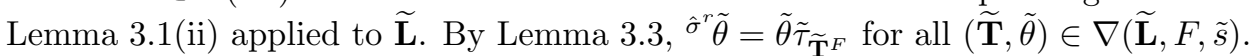

Let $c$ be a block of $\mathcal{O} \mathbf{L}^{F}$ covered by $b^{\prime}$, and let $\chi \in \operatorname{Irr}(c) \cap \mathcal{E}\left(\mathbf{L}^{F}, s\right)$. Then since $C_{\mathbf{L}^{*}}^{\circ}(s)$ has all classical components, it follows from Lemma 3.8, applied to $\mathbf{L}$, that there exists an $x \in \widetilde{\mathbf{L}}^{F}$ such that

$$
\hat{\sigma}^{r} \chi={ }^{x}\left(\chi \tilde{\tau}_{\mathbf{L}^{F}}\right) .
$$

Let $t:=s^{\ell^{r}-1}=i^{*}(\tilde{t})$. Since $i^{*}$ is a surjective homomorphism and $\tilde{t}$ is central, $t \in$ $Z\left(\mathbf{L}^{*}\right)$. Further, by Lemma 3.4, $\tau:=\tilde{\tau}_{\mathbf{L}^{F}}$ is the linear character of $\mathbf{L}^{F}$ corresponding 
to $t$. Since $N^{*}$ centralises $s, N^{*}$ centralises $t$. Hence by Lemma 3.1 and by (2), $\tau$ is $N^{F}$-stable. Since $N^{F} / \mathbf{L}^{F}$ is cyclic and of $\ell^{\prime}$-order, $\tau$ extends to an irreducible character $\check{\tau}$ of $N^{F}$ of $\ell^{\prime}$-order. Note that $\check{\tau}$ takes values in $\mathcal{O}$.

Let $\check{\chi} \in \operatorname{Irr}\left(N^{F} \mid \chi\right) \cap \operatorname{Irr}\left(b^{\prime}\right)$, and let $d$ be the block of $\mathcal{O} N^{F}$ containing $\check{\chi} \check{\tau}$. Since $\widetilde{\mathbf{L}}^{F}$ acts on $N^{F}$ by Lemma 4.3. both ${ }^{\hat{\sigma}^{r}} \check{\chi}$ and ${ }^{x} \check{\chi}^{x} \check{\tau}$ are elements of $\operatorname{Irr}\left(N^{F} \mid \hat{\sigma}^{r} \chi\right)=$ $\operatorname{Irr}\left(N^{F} \mid{ }^{x} \chi^{x} \tau\right)$. Also, ${ }^{x} d$ is the block of $\mathcal{O} N^{F}$ containing ${ }^{x} \check{\chi}^{x} \tilde{\tau}$. Since $N^{F} / \mathbf{L}^{F}$ is cyclic and hence abelian, it follows from Lemma 2.9 that there is an isomorphism $\mathcal{O} N^{F} \hat{\sigma}^{r}\left(b^{\prime}\right) \cong \mathcal{O} N^{F x} d$ which restricts to the identity on $Z \leq Z\left(\mathbf{L}^{F}\right)$. Conjugation by $x^{-1}$ induces an isomorphism $\mathcal{O} N^{F x} d \cong \mathcal{O} N^{F} d$ which is the identity on $Z \leq \mathbf{G}^{F}$. Finally, the $\mathcal{O}$-algebra automorphism $\mathcal{O} N^{F} \rightarrow \mathcal{O} N^{F}$ satisfying $n \mapsto \check{\tau}\left(n^{-1}\right) n$ for all $n \in \mathbf{N}^{F}$ restricts to an isomorphism $\mathcal{O} N^{F} d \cong \mathcal{O} N^{F} b^{\prime}$. This last isomorphism also restricts to the identity on $Z$ since $\check{\tau}$ is an $\ell^{\prime}$-character and $Z$ is an $\ell$-group. Thus $\mathcal{O} N^{F} \hat{\sigma}^{r}\left(b^{\prime}\right) \cong \mathcal{O} N^{F} b^{\prime}$ via an isomorphism which is the identity on $Z$.

Hence $\mathcal{O}\left(N^{F} / Z\right) \overline{\hat{\sigma}^{r}\left(b^{\prime}\right)} \cong \mathcal{O}\left(N^{F} / Z\right) \bar{b}^{\prime}$, where $\bar{b}^{\prime}$ denotes the block of $\mathcal{O}\left(N^{F} / Z\right)$ dominated by $b^{\prime}$. The result then follows by Lemma 2.10(iv).

The next result is similar in spirit to the previous one and will be used to deal with some cases where $C_{\mathbf{L}^{*}}^{\circ}(s)$ has an exceptional component.

Theorem 4.5. Keep the notation and hypothesis of Proposition 4.2. Suppose that $s \in Z\left(\mathbf{L}^{*}\right)^{F}$ and $a \in \mathbb{N}$ is such that ${ }^{\hat{\sigma}^{a}} \chi^{\prime}=\chi^{\prime}$ for all unipotent characters $\chi^{\prime} \in$ $\operatorname{Irr}\left(\mathbf{L}^{F}\right)$. Then

$$
\mathcal{O}\left(N^{F} / Z\right) \hat{\sigma}^{a}\left(\overline{b^{\prime}}\right) \cong \mathcal{O}\left(N^{F} / Z\right) \overline{b^{\prime}} .
$$

Proof. Let $\psi \in \operatorname{Irr}\left(b^{\prime}\right)$ and $\chi \in \mathcal{E}\left(\mathbf{L}^{F}, s\right)$ such that $\psi$ covers $\chi$. Let $\hat{s}$ denote the linear character of $\mathbf{L}^{F}$ corresponding to $s$ as in Lemma 3.1)(ii). Note that $\hat{s}$ is $N^{F}$. stable. By [13, Proposition 13.30, (ii)] there exists a unipotent character $\chi^{\prime}$ of $\mathbf{L}^{F}$ (necessarily unique) such that $\chi=\hat{s} \chi^{\prime}$. By assumption, therefore ${ }^{\hat{\sigma}^{a}} \chi=\hat{\sigma}^{a}\left(\hat{s} \chi^{\prime}\right)=$ $\left(\hat{\sigma}^{a} \hat{s}\right) \chi^{\prime}$. Let $\xi=\hat{\sigma}^{a}(\hat{s}) \hat{s}^{-1}$. Then ${ }^{\hat{\sigma}^{a}} \chi=\xi \chi$.

Since $\xi$ is a linear $N^{F}$-stable character of $\mathbf{L}^{F}$ and $N^{F} / \mathbf{L}^{F}$ is cyclic by assumption, $\xi$ extends to a linear character $\hat{\xi} \in \operatorname{Irr}\left(N^{F}\right)$. Hence ${ }^{\hat{\sigma}^{a}} \psi$ and $\hat{\xi} \psi$ are both elements of $\operatorname{Irr}\left(\left.N^{F}\right|^{\sigma^{a}} \chi\right)=\operatorname{Irr}\left(N^{F} \mid \xi \chi\right)$. Let $d$ be the block of $\mathcal{O} N^{F}$ containing $\hat{\xi} \psi$. Since $N^{F} / \mathbf{L}^{F}$ is abelian, it follows from Lemma 2.9 that there is an isomorphism $\mathcal{O} N^{F} \hat{\sigma}^{a}\left(b^{\prime}\right) \cong \mathcal{O} N^{F} d$ which restricts to the identity on $Z \leq Z\left(\mathbf{L}^{F}\right)$. As in Theorem 4.4, there is an isomorphism $\mathcal{O} N^{F} d \cong \mathcal{O} N^{F} b^{\prime}$ which restricts to the identity on $Z \leq Z\left(\mathbf{L}^{F}\right)$. Composition induces an isomorphism $\mathcal{O} N^{F} \hat{\sigma}^{a}\left(b^{\prime}\right) \cong \mathcal{O} N^{F} b^{\prime}$ which restricts to the identity on $Z \leq Z\left(\mathbf{L}^{F}\right)$.

Remark 4.6. Here and in Section 4, we have assumed that $F$ is a Frobenius morphism. However, suitable analogues of the results of these sections, in particular Theorem 4.1 hold under the weaker assumption that some power of $F$ is a Frobenius morphism, and thus may also be applied to the Ree and Suzuki groups. We will make use of this in Proposition 6.2. We have chosen to stick to the Frobenius case for the general exposition as most of our references for these sections make this assumption.

\section{Blocks OF Finite GROUPS OF LiE TYPE IN NONDEFINING CHARACTERISTIC}

We keep the notation of Section 4 In addition, we assume in this section that $\mathbf{G}$ is simple and simply connected. Throughout $b$ will denote a block of $\mathcal{O G}^{F}$ in $\mathcal{E}_{\ell}\left(\mathbf{G}^{F}, s\right), Z$ an $\ell$-subgroup of $Z\left(\mathbf{G}^{F}\right)$, and $\bar{b}$ the block of $\mathcal{O}\left(\mathbf{G}^{F} / Z\right)$ dominated 
by $b$. Further, whenever $N^{F} / \mathbf{L}^{F}$ is cyclic we will denote by $b^{\prime}$ the block of $\mathcal{O} N^{F}$ in bijection with $b$ as in Theorem 4.1 and $\bar{b}^{\prime}$ will denote the block of $\mathcal{O}\left(N^{F} / Z\right)$ dominated by $b^{\prime}$.

Our first result lifts some of the results of [19] on unipotent blocks to $\mathcal{O}$. We note that the character theoretic arguments applied to blocks of $k \mathbf{G}^{F}$ in 19 also apply to blocks of $\mathcal{O} \mathbf{G}^{F}$. In particular, if $b$ is a block of $\mathcal{O} \mathbf{G}^{F}$ containing characters whose sum is rational valued (that is, there exist $\chi_{1}, \ldots, \chi_{r} \in \operatorname{Irr}(b)$ such that $\left(\chi_{1}+\cdots+\chi_{r}\right)(g) \in \mathbb{Q}$ for all $\left.g \in G\right)$, then $\hat{\sigma}(b)=b$.

Proposition 5.1. Suppose that $s=1$. Then $\mathcal{O}\left(\mathbf{G}^{F} / Z\right) \hat{\sigma}^{r}(\bar{b}) \cong \mathcal{O}\left(\mathbf{G}^{F} / Z\right) \bar{b}$ with $r=1,2$ and if $\mathbf{G}$ is not of type $E_{7}$ or $E_{8}, \mathcal{O}\left(\mathbf{G}^{F} / Z\right) \hat{\sigma}(\bar{b}) \cong \mathcal{O}\left(\mathbf{G}^{F} / Z\right) \bar{b}$.

Proof. The unipotent characters of classical groups are determined by their uniform projections [34, 8.1(a)] and the characters $R_{\mathbf{T}}^{\mathbf{G}}(1)$ are rational valued, hence the unipotent characters of classical groups are rational valued. So if $\mathbf{G}$ is of classical type, then $\hat{\sigma}(b)=b$. Assume now that $\mathbf{G}$ is of exceptional type, and let $b=$ $b_{\mathbf{G}^{F}}(\mathbf{L}, \lambda)$ for a unipotent $e$-cuspidal pair $(\mathbf{L}, \lambda)$ of $\ell$-central defect (see [8, Theorem 4.4] and [18, Théorème $\mathrm{A}]$ ). If $\mathbf{G}=\mathbf{L}$, then $b$ has cyclic defect groups (see the proof of [19, Theorem 5.5]), so by Proposition 2.6 we may assume that $\mathbf{L}$ is a proper Levi subgroup of $\mathbf{G}$.

If $\mathbf{G}$ is of type $E_{7}$ or $E_{8}$, then by [22, Table 1], ${ }^{\hat{\sigma}^{r}} \lambda=\lambda$ for some $r \leq 2$. Hence by the proof of [19, Lemma 5.2], $\hat{\sigma}^{r}(b)=b$, and therefore $\mathcal{O} \mathbf{G}^{F} \hat{\sigma}^{r}(b) \cong \mathcal{O} \mathbf{G}^{F} b$ via an isomorphism which is trivial on $Z$. Thus by Lemma 2.10(iv), $\mathcal{O}\left(\mathbf{G}^{F} / Z\right) \hat{\sigma}^{r}(\bar{b}) \cong$ $\mathcal{O}\left(\mathbf{G}^{F} / Z\right) \bar{b}$ for some $r \leq 2$. If $\mathbf{G}$ is not of type $E_{7}$ or $E_{8}$, then ${ }^{\hat{\sigma}} \lambda=\lambda$ by [22, Table $1]$, and hence, by the same arguments, $\mathcal{O}\left(\mathbf{G}^{F} / Z\right) \hat{\sigma}(\bar{b}) \cong \mathcal{O}\left(\mathbf{G}^{F} / Z\right) \bar{b}$.

Recall that by [13, Lemma 13.14 (iii)], $C_{\mathbf{G}^{*}}(s) / C_{\mathbf{G}^{*}}^{\circ}(s)$ is isomorphic to a subgroup of $\operatorname{Irr}\left(Z(\mathbf{G}) / Z^{\circ}(\mathbf{G})\right) \cong \operatorname{Irr}(Z(\mathbf{G})) \cong Z(\mathbf{G})$. Further, $Z(\mathbf{G})$ is cyclic in all cases except possibly when $\mathbf{G}$ is of type $D_{m}$ with $m$ even (see [35, Table 24.2]). Therefore $C_{\mathbf{G}^{*}}(s)^{F} / C_{\mathbf{G}^{*}}^{\circ}(s)^{F}$, and hence $N^{F} / \mathbf{L}^{F}$, is cyclic in all cases except possibly when $\mathbf{G}$ is of type $D_{m}$ with $m$ even. We will use this fact without further comment. Also, we note that in many cases below $Z=1$.

Recall that a semisimple element $t$ of a connected reductive group $\mathbf{H}$ is called isolated if its connected centraliser $C_{\mathbf{H}}^{\circ}(t)$ is not contained in any proper Levi subgroup of $\mathbf{H}$. The element $t$ is isolated in $\mathbf{H}$ if and only if the image, $\bar{t}$, of $t$ in $\mathbf{H} / Z(\mathbf{H})$ is isolated in $\mathbf{H} / Z(\mathbf{H})$. The quotient $\mathbf{H} / Z(\mathbf{H})$ is a direct product of adjoint simple groups and $\bar{t}$ is isolated in $\mathbf{H} / Z(\mathbf{H})$ if and only if the projection of $\bar{t}$ on every simple factor of $\mathbf{H} / Z(\mathbf{H})$ is isolated. Suppose that $F^{\prime}: \mathbf{H} \rightarrow \mathbf{H}$ is a Frobenius morphism. Then if $t \in \mathbf{H}^{F^{\prime}}, a_{\mathbf{H}^{F^{\prime}}}(t)$ is a divisor of the order of $\bar{t}$ in $\mathbf{H} / Z(\mathbf{H})$. Thus, by the classification of isolated elements in simple algebraic groups, [3, Section 5] (see also [40, Table 6.2]), if $t \in \mathbf{H}^{F^{\prime}}$ is an isolated $\ell^{\prime}$-element of $\mathbf{H}$ and $\mathbf{H}$ has all classical components, then $a_{\mathbf{H}^{F^{\prime}}}(t)$ equals 1 or 2 , and if $\mathbf{H}$ has at most one component not of type A, then $a_{\mathbf{H}^{F^{\prime}}}(t) \leq 6$. We note that Table 2 of 3 lists an isolated element of order 4 in type $D$, but the listed element is in fact of order 2 .

By choice of $\mathbf{L}^{*}, s$ is isolated in $\mathbf{L}^{*}$, and whenever $s$ is isolated in $\mathbf{G}^{*}$, then $N^{*}=\mathbf{L}^{*}=\mathbf{G}^{*}$ and $b=b^{\prime}$.

Proposition 5.2. Suppose that $\mathbf{G}$ is of type $A, B$, or $C$. Then $\mathcal{O}\left(\mathbf{G}^{F} / Z\right) \bar{b}$ is Morita equivalent to $\mathcal{O}\left(N^{F} / Z\right) \bar{b}^{\prime}$ and $\mathcal{O}\left(N^{F} / Z\right) \bar{b}^{\prime} \cong \mathcal{O}\left(N^{F} / Z\right) \hat{\sigma}\left(\bar{b}^{\prime}\right)$. 
Proof. Since $\mathbf{L}^{*}$ is a classical group, $a_{\mathbf{L}^{* F}}(s) \leq 2$ for all isolated elements $s \in \mathbf{L}^{* F}$. Since $s$ is an $\ell^{\prime}$-element, $a_{\mathbf{L}^{* F}}(s)=2$ can only occur when $\ell$ is odd. Hence $r_{\mathbf{L}^{* F}}(s)=$ 1. Thus the result follows from Proposition 4.2 and Theorem 4.4 .

Proposition 5.3. Suppose that $\mathbf{G}$ is of type D.

(i) If $N^{F} / \mathbf{L}^{F}$ is cyclic, then $\mathcal{O}\left(\mathbf{G}^{F} / Z\right) \bar{b}$ is Morita equivalent to $\mathcal{O}\left(N^{F} / Z\right) \bar{b}^{\prime}$ and $\mathcal{O}\left(N^{F} / Z\right) \bar{b}^{\prime} \cong \mathcal{O}\left(N^{F} / Z\right) \hat{\sigma}\left(\bar{b}^{\prime}\right)$.

(ii) If $N^{F} / \mathbf{L}^{F}$ is not cyclic, then $\mathcal{O}\left(\mathbf{G}^{F} / Z\right) \hat{\sigma}^{r}(\bar{b}) \cong \mathcal{O}\left(\mathbf{G}^{F} / Z\right) \bar{b}$ for some $r=$ 1,2 .

Proof. The proof of part (i) is identical to that of Proposition 5.2. Suppose that $N^{F} / \mathbf{L}^{F}$ is not cyclic. Then $N^{F} / \mathbf{L}^{F} \cong C_{2} \times C_{2}$. Further, $\mathbf{G}^{F}=\operatorname{Spin}_{2 n}^{+}(q)$ and $s$ is a quasi-isolated element of $\mathbf{G}^{* F}$ of order 4 such that $C_{\mathbf{G}^{*}}^{\circ}(s)$ is of type $A_{2 n-3}$ (see [7, Remark 9.24]). Since $N^{F} / \mathbf{L}^{F} \subseteq\left(C_{\mathbf{G}^{*}}(s) / C_{\mathbf{G}^{*}}^{\circ}(s)\right)^{F}$ and $\exp \left(C_{\mathbf{G}^{*}}(s) / C_{\mathbf{G}^{*}}^{\circ}(s)\right)=$ 2 divides the order of the $\ell^{\prime}$-element $s$ [13, Remark 13.15 (i)], $\ell$ is odd. Let $r=$ $r_{\mathbf{G}^{* F}}(s)$ and note that $r \leq 2$.

Let $i: \mathbf{G} \hookrightarrow \widetilde{\mathbf{G}}$ be a regular embedding, and let $\tilde{s} \in \widetilde{\mathbf{G}}^{* F}$ be a semisimple $\ell^{\prime}$ element such that $i^{*}(\tilde{s})=s$. It follows from Lemma 3.3(ii) that ${ }^{\hat{\sigma}^{r}} \tilde{\theta}=\tilde{\theta}_{\widetilde{\mathbf{T}}^{F}}$ for all $(\widetilde{\mathbf{T}}, \tilde{\theta}) \in \nabla(\widetilde{\mathbf{G}}, F, \tilde{s})$, where $\tilde{\tau}$ is the linear character of $\widetilde{\mathbf{G}}^{F}$ corresponding to $\tilde{s}^{\ell^{r}-1}$.

Suppose that $\chi \in \operatorname{Irr}(b) \cap \mathcal{E}\left(\mathbf{G}^{F}, s\right)$. By Lemma 3.8, since $\mathbf{G}$ is of classical type, $\hat{\sigma}^{r} \chi={ }^{x}\left(\chi \tilde{\tau}_{\mathbf{G}^{F}}\right)$ for some $x \in \widetilde{\mathbf{G}}^{F}$. Let $d$ be the block of $\mathcal{O} \mathbf{G}^{F}$ containing $\chi \tilde{\tau}_{\mathbf{G}^{F}}$. Then $\hat{\sigma}^{r}(b)$ is the block of $\mathcal{O} \mathbf{G}^{F}$ containing ${ }^{x}\left(\chi \tilde{\tau}_{\mathbf{G}^{F}}\right)$, so $\hat{\sigma}^{r}(b)={ }^{x} d$. Conjugation by $x^{-1}$ induces an isomorphism $\mathcal{O} \mathbf{G}^{F x} d \cong \mathcal{O} \mathbf{G}^{F} d$ which is the identity on $Z \leq \mathbf{G}^{F}$. The automorphism $\mathcal{O G}^{F} \rightarrow \mathcal{O} \mathbf{G}^{F}$ given by $g \mapsto \tilde{\tau}_{\mathbf{G}^{F}}\left(g^{-1}\right) g$ induces an isomorphism $\mathcal{O} \mathbf{G}^{F} d \cong \mathcal{O} \mathbf{G}^{F} b$ which is also trivial on $Z$. Hence, $\mathcal{O G}^{F} \hat{\sigma}^{r}(b) \cong \mathcal{O} \mathbf{G}^{F} b$ via an isomorphism which is trivial when restricted to $Z$ yielding an isomorphism $\mathcal{O}\left(\mathbf{G}^{F} / Z\right) \hat{\sigma}^{r}(\bar{b}) \cong \mathcal{O}\left(\mathbf{G}^{F} / Z\right) \bar{b}$.

Proposition 5.4. Suppose that $\mathbf{G}$ is of type $G_{2}, F_{4}$, or $E_{6}$ and $s \neq 1$. Then $\mathcal{O}\left(\mathbf{G}^{F} / Z\right) \bar{b}$ is Morita equivalent to $\mathcal{O}\left(N^{F} / Z\right) \bar{b}^{\prime}$. If $s$ is isolated in $\mathbf{G}^{*}$ and either $o(s)=3$ and $\ell \equiv 2 \bmod 3$, or $o(s)=4$ and $\ell \equiv 3 \bmod 4$, then $\mathcal{O}\left(N^{F} / Z\right) \bar{b}^{\prime} \cong$ $\mathcal{O}\left(N^{F} / Z\right) \hat{\sigma}^{r}\left(\bar{b}^{\prime}\right)$ for some $r=1,2$. Otherwise, $\mathcal{O}\left(N^{F} / Z\right) \bar{b}^{\prime} \cong \mathcal{O}\left(N^{F} / Z\right) \hat{\sigma}\left(\bar{b}^{\prime}\right)$.

Proof. The first assertion is Proposition 4.2, If $s$ is isolated in $\mathbf{G}^{*}$ and either $o(s)=3$ and $\ell \equiv 2 \bmod 3$, or $o(s)=4$ and $\ell \equiv 3 \bmod 4$, then $r_{\mathbf{L}^{* F}}(s)=2$. Otherwise $r_{\mathbf{L}^{* F}}(s)=1$. Since for all nontrivial semisimple $\ell^{\prime}$-elements $s \in \mathbf{G}^{* F}, C_{\mathbf{L}^{*}}^{\circ}(s)$ has all classical components by [3, Section 5], the result follows from Theorem 4.4.

Proposition 5.5. Suppose that $\mathbf{G}$ is of type $E_{7}$ and $s \neq 1$. Then $\mathcal{O}\left(\mathbf{G}^{F} / Z\right) \bar{b}$ is Morita equivalent to $\mathcal{O}\left(N^{F} / Z\right) \bar{b}^{\prime}$. If $\ell \equiv 2 \bmod 3$ and one of the following holds,

- $s$ is isolated in $\mathbf{G}^{*}$ and $o(s)=3$,

- $s$ is not isolated in $\mathbf{G}^{*}, s \in Z\left(\mathbf{L}^{*}\right)^{F}$ and $\mathbf{L}^{*}$ has a component of type $E_{6}$,

- $s$ is not isolated in $\mathbf{G}^{*}, \mathbf{L}^{*}$ has a component of type $E_{6}$ and $a_{\mathbf{L}^{*}}(s)=3$,

or if $\ell \equiv 3 \bmod 4$ and $s$ is isolated in $\mathbf{G}^{*}$ with $o(s)=4$, then $\mathcal{O}\left(N^{F} / Z\right) \bar{b}^{\prime} \cong$ $\mathcal{O}\left(N^{F} / Z\right) \hat{\sigma}^{r}\left(\bar{b}^{\prime}\right)$ for some $r=1,2$. Otherwise, $\mathcal{O}\left(N^{F} / Z\right) \bar{b}^{\prime} \cong \mathcal{O}\left(N^{F} / Z\right) \hat{\sigma}\left(\bar{b}^{\prime}\right)$.

Proof. The first assertion is just Proposition 4.2. Suppose that $s$ is isolated in $\mathbf{G}^{*}$. Then if $o(s)=3$ and $\ell \equiv 2 \bmod 3$ or if $o(s)=4$ and $\ell \equiv 3 \bmod 4, r_{\mathbf{G}^{* F}}(s)=2$. Otherwise $r_{\mathbf{G}^{* F}}(s)=1$. Since $C_{\mathbf{G}^{*}}^{\circ}(s)$ has all classical components by [3, Section $5]$, the result follows by applying Theorem 4.4 with $\mathbf{L}^{*}=N^{*}=\mathbf{G}^{*}$. 
Now suppose that $s$ is not isolated in $\mathbf{G}^{*}$ and $s \in Z\left(\mathbf{L}^{*}\right)^{F}$. If $\ell \equiv 2 \bmod 3$ and $\mathbf{L}^{*}$ has a component of type $E_{6}$, then the minimal $a$ such that ${ }^{\hat{\sigma}^{a}} \chi^{\prime}=\chi^{\prime}$ for all unipotent characters $\chi^{\prime}$ of $\operatorname{Irr}\left(\mathbf{L}^{F}\right)$ is 2 . Otherwise the minimal such $a$ is 1 , so the result follows from Theorem 4.5 .

Finally, suppose that $s$ is not isolated in $\mathbf{G}^{*}$ and $s \notin Z\left(\mathbf{L}^{*}\right)^{F}$. If $\ell \equiv 2 \bmod 3$, $\mathbf{L}^{*}$ has a component of type $E_{6}$, and $a_{\mathbf{L}^{*}}(s)=3$, then $r_{\mathbf{L}^{* F}}(s)=2$. Otherwise, $r_{\mathbf{L}^{* F}}(s)=1$. Again, since $C_{\mathbf{L}^{*}}^{\circ}(s)$ has all classical components by [3, Section 5], the result follows from Theorem 4.4 .

There are three nontrivial isolated elements in $E_{8}$ whose centralisers have an exceptional component. These cases are dealt with separately in Section 5.1 .

Proposition 5.6. Suppose that $\mathbf{G}$ is of type $E_{8}, s \neq 1$, and that if $s$ is isolated in $\mathbf{G}^{*}$, then $C_{\mathbf{G}^{*}}(s)$ has all classical components. The block $\mathcal{O}\left(\mathbf{G}^{F} / Z\right) \bar{b}$ is Morita equivalent to $\mathcal{O}\left(N^{F} / Z\right) \bar{b}^{\prime}$.

- If $s$ is isolated in $\mathbf{G}^{*}, o(s)=5$, and $\ell \equiv 2$ or $3 \bmod 5$, then $\mathcal{O}\left(N^{F} / Z\right) \bar{b}^{\prime} \cong$ $\mathcal{O}\left(N^{F} / Z\right) \hat{\sigma}^{r}\left(\bar{b}^{\prime}\right)$ for some $r \leq 4$.

- If one of the following holds,

$-s$ is isolated in $\mathbf{G}^{*}, o(s)=3$, and $\ell \equiv 2 \bmod 3 ;$ or $o(s)=4$ and $\ell \equiv 3$ $\bmod 4$; or $o(s)=5$ and $\ell \equiv 4 \bmod 5$; or $o(s)=6$ and $\ell \equiv 5 \bmod 6$,

- $s$ is not isolated in $\mathbf{G}^{*}, s \in Z\left(\mathbf{L}^{*}\right)^{F}$, $\mathbf{L}^{*}$ has a component of type $E_{6}$ or $E_{7}$, and $\ell \equiv 2 \bmod 3$,

- $s$ is not isolated in $\mathbf{G}^{*}, s \notin Z\left(\mathbf{L}^{*}\right)^{F}, \mathbf{L}^{*}$ has a component of type $E_{7}$, $a_{\mathbf{L}^{*}}(s)=4$, and $\ell \equiv 3 \bmod 4$, or

- $s$ is not isolated in $\mathbf{G}^{*}, s \notin Z\left(\mathbf{L}^{*}\right)^{F}$, $\mathbf{L}^{*}$ has a component of type $E_{6}$ or $E_{7}, a_{\mathbf{L}^{*}}(s)=3$, and $\ell \equiv 2 \bmod 3$,

then $\mathcal{O}\left(N^{F} / Z\right) \bar{b}^{\prime} \cong \mathcal{O}\left(N^{F} / Z\right) \hat{\sigma}^{r}\left(\bar{b}^{\prime}\right)$ for some $r=1,2$.

- In all other cases $\mathcal{O}\left(N^{F} / Z\right) \bar{b}^{\prime} \cong \mathcal{O}\left(N^{F} / Z\right) \hat{\sigma}\left(\bar{b}^{\prime}\right)$.

Proof. First suppose that $s$ is isolated in $\mathbf{G}^{*}$. If $o(s)=5$ and $\ell \equiv 2$ or $3 \bmod 5$, then $r_{\mathbf{G}^{* F}}(s)=4$; if $o(s)=3$ and $\ell \equiv 2 \bmod 3$, or $o(s)=4$ and $\ell \equiv 3 \bmod 4$, or $o(s)=5$ and $\ell \equiv 4 \bmod 5$, or $o(s)=6$ and $\ell \equiv 5 \bmod 6$, then $r_{\mathrm{G}^{* F}}(s)=2$; otherwise $r_{\mathbf{G}^{* F}}(s)=1$. As we are assuming that $C_{\mathbf{G}^{*}}(s)$ has all classical components, we can then apply Theorem 4.4 .

Now suppose that $s$ is not isolated in $\mathbf{G}^{*}$ and $s \in Z\left(\mathbf{L}^{*}\right)^{F}$. If $\ell \equiv 2 \bmod 3$ and $\mathbf{L}^{*}$ has a component of type $E_{6}$ or $E_{7}$, then the minimal $a$ such that ${ }^{\hat{\sigma}^{a}} \chi^{\prime}=\chi^{\prime}$ for all unipotent characters $\chi^{\prime}$ of $\operatorname{Irr}\left(\mathbf{L}^{F}\right)$ is 2 . Otherwise the minimal such $a$ is 1 , and the result follows from Theorem 4.5 .

Finally, suppose that $s$ is not isolated in $\mathbf{G}^{*}$ and $s \notin Z\left(\mathbf{L}^{*}\right)^{F}$. If $\mathbf{L}^{*}$ has a component of type $E_{7}, \ell \equiv 3 \bmod 4$, and $a_{\mathbf{L}^{*}}(s)=4$, or if $\mathbf{L}^{*}$ has a component of type $E_{6}$ or $E_{7}, \ell \equiv 2 \bmod 3$, and $a_{\mathbf{L}^{*}}(s)=3$, then $r_{\mathbf{L}^{* F}}(s)=2$. Otherwise, $r_{\mathbf{L}^{* F}}(s)=1$. Thus since $C_{\mathbf{L}^{*}}^{\circ}(s)$ has all classical components, the result follows from Theorem 4.4 .

5.1. Isolated blocks of $E_{8}$ with $C_{\mathbf{G}^{*}}(s)$ of nonclassical type. In this subsection we assume that $\mathbf{G}=E_{8}$. We will deal with the nontrivial isolated semisimple elements $s \in \mathbf{G}^{* F}$ which are not covered by Proposition 5.6.

Notation 5.7. We let $\mathcal{C}_{b}$ denote the set of blocks in $\mathcal{E}_{\ell}\left(\mathbf{G}^{F}, s\right)$ which are Galois conjugates of $b$, that is, blocks of the form $\hat{\sigma}^{m}(b), m \in \mathbb{N}$. 
Lemma 5.8. Set $\left|\mathcal{C}_{b}\right|=m$ and $r=r_{\mathbf{G}^{* F}}(s)$. Then $\hat{\sigma}^{r}(b)$ is a block in $\mathcal{E}_{\ell}\left(\mathbf{G}^{F}, s\right)$ and $b=\hat{\sigma}^{n}(b)$ for some $n \leq r m$.

Proof. By Lemma 3.6(ii) the action of the group $\langle\hat{\sigma}\rangle$ on $\operatorname{Irr}\left(\mathbf{G}^{F}\right)$ induces an action on the set of Lusztig series $\mathcal{E}\left(\mathbf{G}^{F}, t\right)$, where $t$ runs over the $\mathbf{G}^{* F}$-conjugacy classes of $\ell^{\prime}$-elements of $\mathbf{G}^{* F}$. Further, since $Z\left(\mathbf{G}^{* F}\right)=1, a_{\mathbf{G}^{* F}}(s)=o(s)$, and it follows that $s^{\ell^{r}}=s$. Thus the stabiliser in $\langle\hat{\sigma}\rangle$ of $\mathcal{E}\left(\mathbf{G}^{F}, s\right)$ is of the form $\left\langle\hat{\sigma}^{u}\right\rangle$ for some nonnegative integer $u$ dividing $r$. In particular, the first assertion is proved. Now, the set of $\ell$-blocks of $\mathbf{G}^{F}$ contained in $\mathcal{E}_{\ell}\left(\mathbf{G}^{F}, s\right)$ is $\left\langle\hat{\sigma}^{u}\right\rangle$-invariant and $\mathcal{C}_{b}$ is the $\left\langle\hat{\sigma}^{u}\right\rangle$-orbit of $b$ under the action of $\left\langle\hat{\sigma}^{u}\right\rangle$ on this set; hence $b=\hat{\sigma}^{u m}(b)$, proving the second assertion.

Proposition 5.9. Let $\mathbf{G}$ be a simple algebraic group of type $E_{8}$ and suppose that $1 \neq s \in \mathbf{G}^{* F}$ is an isolated semisimple $\ell^{\prime}$-element such that $C_{\mathbf{G}^{*}}(s)$ has an exceptional component. Then $o(s)=2$ or $o(s)=3$. If $o(s)=3$, then $\mathcal{O G}^{F} b \cong \mathcal{O} \mathbf{G}^{F} \hat{\sigma}^{r}(b)$ for some $r \leq 4$, and if $o(s)=2$, then $\mathcal{O G}^{F} b \cong \mathcal{O} \mathbf{G}^{F} \hat{\sigma}^{r}(b)$ for some $r \leq 2$.

Proof. By [30, Table 1, Table 5], it is enough to consider $s$ in the following cases.

\begin{tabular}{c|c}
$o(s)$ & Components of $C_{\mathbf{G}^{*}}(s)^{F}$ \\
\hline \hline 2 & $E_{7} \times A_{1}$ \\
3 & $E_{6} \times A_{2}$ \\
3 & ${ }^{2} E_{6} \times{ }^{2} A_{2}$
\end{tabular}

In particular, the first assertion of the proposition holds. If $b$ has cyclic defect groups, then the remaining assertion follows from Proposition 2.6. For the rest of the proof we assume that $b$ has noncyclic defect groups. If $o(s)=2$, then $r_{\mathbf{G}^{* F}}(s)=1$, and if $o(s)=3$, then $r_{\mathbf{G}^{* F}}(s)=2$. Thus, by Lemma 5.8 it suffices to prove that $\left|\mathcal{C}_{b}\right| \leq 2$. Let $\mathbf{G}^{F}=E_{8}(q)$, and if $\ell$ is odd, let $e$ be the order of $q$ modulo $\ell$. If $\ell=2$, let $e$ be the order of $q$ modulo 4 . We will use the parametrisation of $\ell$-blocks of $\mathbf{G}^{F}$ by $e$-cuspidal pairs to obtain the desired bound on $\left|\mathcal{C}_{b}\right|$.

First suppose that $\ell \leq 5$ (i.e., $\ell$ is bad for $\mathbf{G}$ ). By [30, Theorem 1.2], the blocks in $\mathcal{E}_{\ell}\left(\mathbf{G}^{F}, s\right)$ are in bijection with $\mathbf{G}^{F}$-conjugacy classes of pairs $(\mathbf{M}, \lambda)$ of $\mathbf{G}$ such that $(\mathbf{M}, \lambda)$ is an $e$-cuspidal pair of $\mathbf{G}$, and $\lambda \in \mathcal{E}\left(\mathbf{M}^{F}, s\right)$ is of quasi-central $\ell$ defect. The bijection is described via Lusztig induction: a block corresponds to a pair $(\mathbf{M}, \lambda)$ if and only if all irreducible constituents of $R_{\mathbf{M}}^{\mathbf{G}}(\lambda)$ lie in the block. The tables in Section 6 of 30 list the $e$-cuspidal pairs of G. Since $\hat{\sigma}$ commutes with Lusztig induction, if the pair $(\mathbf{M}, \lambda)$ corresponds to the block $b$, then $\left(\mathbf{M},{ }^{{ }^{m}} \lambda\right)$ corresponds to $\hat{\sigma}^{m}(b)$ for $\hat{\sigma}^{m}(b)$ in $\mathcal{C}_{b}$. Further, if $g \in \mathbf{G}^{F}$, then ${ }^{g} \lambda=\lambda$ if and only if ${ }^{g}\left(\hat{\sigma}^{m} \lambda\right)=\hat{\sigma}^{m} \lambda$, and hence $(\mathbf{M}, \lambda)$ and $\left(\mathbf{M}, \hat{\sigma}^{m} \lambda\right)$ have the same relative Weyl group. Thus, all blocks in $\mathcal{C}_{b}$ correspond to the same numbered line of the tables in Section 6 of [30]. Moreover, since $\lambda$ and ${ }^{\hat{\sigma}^{m}} \lambda$ have the same degree, by the degree formula for Jordan correspondence (see [13, Remark 13.24]), it follows that if $\mathcal{C}_{b}$ has more than one element, then the $\lambda$ column of the relevant line of the table contains at least two entries of the same degree. Finally, the only relevant lines of the tables are those which correspond to blocks with noncyclic and, in particular, nontrivial defect. Inspection of the tables yields $\left|\mathcal{C}_{b}\right| \leq 2$.

Now suppose that $\ell \geq 7$. In this case block distributions are described in 9 . By [9, Theorem 4.1], the blocks of $\mathbf{G}^{F}$ in $\mathcal{E}_{\ell}\left(\mathbf{G}^{F}, s\right)$ are in bijection with $\mathbf{G}^{F}$ conjugacy classes of pairs $(\mathbf{M}, \lambda)$ of $\mathbf{G}$ such that $(\mathbf{M}, \lambda)$ is an $e$-cuspidal pair of $\mathbf{G}$ 
and with $\lambda \in \mathcal{E}\left(\mathbf{M}^{F}, s\right)$; the bijection is defined by the same condition on Lusztig induction as for bad $\ell$ above. Further, if $(\mathbf{M}, \lambda)$ is an $e$-cuspidal pair for $\mathbf{G}$ and $\alpha \in$ $\mathcal{E}\left(C_{\mathbf{M}^{*}}(s)^{F}, 1\right)$ is in Jordan correspondence with $\lambda$, then $\left(C_{\mathbf{M}^{*}}(s)^{F}, \alpha\right)$ is a unipotent $e$-cuspidal pair for $C_{\mathbf{G}^{*}}(s)$ and the $\ell$-block of $\mathbf{G}^{F}$ corresponding to $(\mathbf{M}, \lambda)$ and the unipotent $\ell$-block of $C_{\mathbf{G}^{*}}(s)^{F}$ corresponding to $\left(C_{\mathbf{M}^{*}}(s)^{F}, \alpha\right)$ have isomorphic defect groups 9, Theorem 4.1, Proposition 5.1]. Now, if the multiplicity of the eth cyclotomic polynomial $\Phi_{e}$ in the polynomial order of $C_{\mathbf{G}^{*}}(s)^{F}$ is at most 1 , then the Sylow $\ell$-subgroups are cyclic, and consequently every $\ell$-block of $C_{\mathbf{G}^{*}}(s)^{F}$ has cyclic defect. Thus, we may assume that this multiplicity is at least 2 ; that is, $e$ is one of $1,2,3,4,6$.

Suppose that $e=1$ or 4 . We refer again to the tables of [30, Section 6] for a list of $e$-cuspidal pairs noting that now unnumbered lines also correspond to blocks. By the same considerations as for the bad primes case it follows that any two blocks in $\mathcal{C}_{b}$ correspond to the same (numbered or unnumbered) line of the table and that if $\left|\mathcal{C}_{b}\right|>1$, then the $\lambda$ column of the relevant line of the table contains at least two entries of the same degree and of positive $\ell$-defect. As before, we obtain $\left|\mathcal{C}_{b}\right| \leq 2$. The results for $e=2$ follow by Ennola duality from the $e=1$ case [6, Section 3A].

Now suppose that $e=3$. The results for $e=6$ will again follow by Ennola duality. The third column of the Table 1 lists pairs $\left(C_{\mathbf{M}^{*}}(s)^{F}, \alpha\right)$ where $\left(C_{\mathbf{M}^{*}}(s), \alpha\right)$ is a unipotent 3-cuspidal pair of $C_{\mathbf{G}^{*}}(s)$. These were calculated using CHEVIE [23] and GAP [38].

Table 1: Unipotent 3-cuspidal pairs of $C_{\mathbf{G}^{*}}(s)$

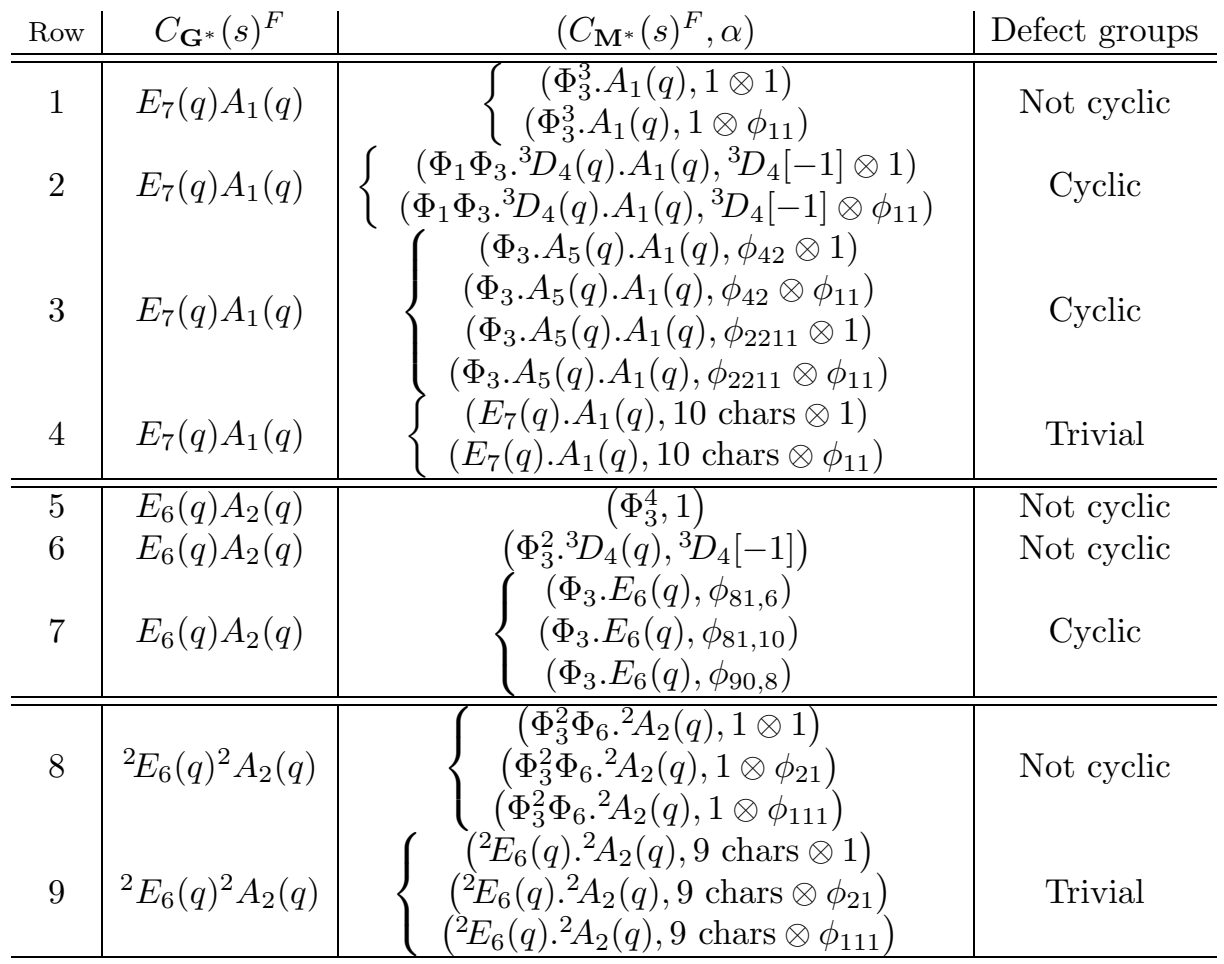


Again, all blocks in $\mathcal{C}_{b}$ correspond to the same line of the table, and the corresponding $\alpha$ 's have the same degree. Since the only relevant lines are those with noncyclic entry in the last column, $\left|\mathcal{C}_{b}\right|=1$.

\section{Defining Characteristic and Ree and Suzuki groups}

In this section $p$ denotes a prime number and $\mathbf{G}$ a simple, simply connected group defined over $\overline{\mathbb{F}}_{p}$. Let $F: \mathbf{G} \rightarrow \mathbf{G}$ be an endomorphism a power of which is a Frobenius morphism, allowing for the case that $\mathbf{G}^{F}$ is a Ree or Suzuki group. We freely use the notation of Sections 3 and 4 in this context.

Proposition 6.1. Suppose that $p=\ell$. Let $b$ be a block of $\mathcal{O G}^{F}$, let $Z \leq Z\left(\mathbf{G}^{F}\right)$, and let $\bar{b}$ be the block of $\mathcal{O}\left(\mathbf{G}^{F} / Z\right)$ dominated by b. Then $\mathcal{O}\left(\mathbf{G}^{F} / Z\right) \hat{\sigma}(\bar{b}) \cong$ $\mathcal{O}\left(\mathbf{G}^{F} / Z\right) \bar{b}$.

Proof. If $Z\left(\mathbf{G}^{F}\right) \leq C_{2}, Z\left(\mathbf{G}^{F}\right) \cong C_{2} \times C_{2}$, or if $b$ is a principal or Steinberg block, then as shown in [19, Theorem 4.1], $\hat{\sigma}(b)=b$.

Suppose that $Z\left(\mathbf{G}^{F}\right) \cong C_{m}$ for some $m>2$ coprime to $\ell$ and assume that $b$ is not the principal or the Steinberg block. Let $\varphi=F_{\ell}^{\phi(m)-1}$ be the group automorphism of $\mathbf{G}^{F}$ defined in [19, Theorem 4.1] with $F_{\ell}$ an $\mathbb{F}_{\ell^{-}}$split Steinberg endomorphism of $\mathbf{G}$ and $\phi$ the Euler totient function. Let $\varphi^{\prime}$ be the $\mathcal{O}$-algebra isomorphism induced by $\varphi$. Then, applying the arguments of [19, Theorem 4.1] to $\varphi^{\prime}$ instead of to the $k$-algebra isomorphism induced by $\varphi$, it follows that $\varphi^{\prime}(b)=\hat{\sigma}(b)$. The restriction $\left.\varphi^{\prime}\right|_{\mathcal{O G}}{ }^{F} b: \mathcal{O} \mathbf{G}^{F} b \rightarrow \mathcal{O} \mathbf{G}^{F} \hat{\sigma}(b)$ is also an $\mathcal{O}$-algebra isomorphism, hence $\mathcal{O} \mathbf{G}^{F} b \cong \mathcal{O} \mathbf{G}^{F} \hat{\sigma}(b)$ as $\mathcal{O}$-algebras.

Now let $Z$ be a central subgroup of $\mathbf{G}^{F}$. Then $Z$ is an $\ell^{\prime}$-group by [35, Table 24.2], so by Lemma 2.10 (ii), $\mathcal{O} \mathbf{G}^{F} b \cong \mathcal{O}\left(\mathbf{G}^{F} / Z\right) \bar{b}$ and $\mathcal{O} \mathbf{G}^{F} \hat{\sigma}(b) \cong \mathcal{O}\left(\mathbf{G}^{F} / Z\right) \overline{\hat{\sigma}(b)}$. Since $\overline{\hat{\sigma}(b)}=\hat{\sigma}(\bar{b})$ by Lemma 2.10 (iv), it follows that $\mathcal{O}\left(\mathbf{G}^{F} / Z\right) \bar{b} \cong \mathcal{O}\left(\mathbf{G}^{F} / Z\right) \hat{\sigma}(\bar{b})$.

The next proposition deals with the Suzuki and Ree groups in nondefining characteristic.

Proposition 6.2. Let $b$ be a block of $\mathcal{O G}^{F}$.

(i) Suppose that $\ell \neq p=2, \mathbf{G}$ is of type $B_{2}$, and $\mathbf{G}^{F}$ is the Suzuki group ${ }^{2} B_{2}\left(2^{2 n+1}\right)$. Then $\mathcal{O} \mathbf{G}^{F} \hat{\sigma}(b) \cong \mathcal{O} \mathbf{G}^{F} b$.

(ii) Suppose that $\ell \neq p=3, \mathbf{G}$ is of type $G_{2}$, and $\mathbf{G}^{F}$ is the Ree group ${ }^{2} G_{2}\left(3^{2 n+1}\right)$. Then $\mathcal{O} \mathbf{G}^{F} \hat{\sigma}(b) \cong \mathcal{O} \mathbf{G}^{F} b$.

(iii) Suppose that $\ell \neq p=2, \mathbf{G}$ is of type $F_{4}$ and $\mathbf{G}^{F}$ is the Ree group ${ }^{2} F_{4}\left(2^{2 n+1}\right)$. There exists an $F$-stable Levi subgroup $\mathbf{L}$ of $\mathbf{G}$ and a block $c$ of $\mathbf{L}^{F}$ such that $\mathcal{O} \mathbf{G}^{F} b$ is Morita equivalent to $\mathcal{O} \mathbf{L}^{F} c$ and $\mathcal{O} \mathbf{L}^{F} c \cong \mathcal{O} \mathbf{L}^{F} \hat{\sigma}^{r}(c)$ for some $r \leq 2$.

Proof. If $b$ is the principal block, then $\hat{\sigma}(b)=b$ and there is nothing to prove. Thus we may assume that $b$ is nonprincipal. First suppose that $\mathbf{G}^{F}$ is as in (i) or (ii). By the proof of [19, Theorem 5.7], $b$ has either cyclic or Klein four defect groups and we are done by Proposition 2.6.

Assume from now on that $\mathbf{G}^{F}$ is of type ${ }^{2} F_{4}\left(2^{2 n+1}\right)$. For $s$ a semisimple element of $\ell^{\prime}$-order of $\mathbf{G}^{* F}, \mathcal{E}_{\ell}\left(\mathbf{G}^{F}, s\right)$ is a union of $\ell$-blocks of $\mathbf{G}^{F}$ (see [25, p. 113]). Moreover, Theorem 4.1 and hence Proposition 4.2 continue to hold with $\mathbf{L}^{*}$ and $\mathbf{L}$ as in Section 5. We note that since $Z(\mathbf{G})=1, N^{F}=\mathbf{L}^{F}$ and $Z=1$.

Let $s \in \mathbf{G}^{* F}$ be a semisimple $\ell^{\prime}$-element such that $b \in \mathcal{E}_{\ell}\left(\mathbf{G}^{F}, s\right)$. If $s=1$, then again by the proof of [19, Theorem 5.7], $b$ has either cyclic or Klein four defect 
groups or $b$ is the principal block, and in these cases we are done as above (with $\mathbf{L}=\mathbf{G}$ ). So we may assume that $s \neq 1$ and hence that $\mathbf{L}$ is a proper Levi subgroup of $\mathbf{G}$. In particular, every simple component of $\mathbf{L}$ and of $C_{\mathbf{G}^{*}}(s)$ has classical type. If $C_{\mathbf{G}^{*}}(s)$ contains no rational component of type ${ }^{2} B_{2}$, then the conclusion of Lemma 3.7 and hence of Theorem 4.4 holds for $s$ and $b$. Further, since all components of $\mathbf{L}$ are of classical type, $a_{\mathbf{L}^{*}}(s) \leq 2$ whence $r_{\mathbf{L}^{*}}(s)=1$. Thus, we obtain $\mathcal{O} \mathbf{L}^{F} c \cong \mathcal{O} \mathbf{L}^{F} \hat{\sigma}(c)$. If $C_{\mathbf{G}^{*}}(s)$ has a rational component of type ${ }^{2} B_{2}$, then $C_{\mathbf{G}^{*}}(s)=\mathbf{L}^{*}$ and the conclusion of Theorem 4.5 holds. Moreover, ${ }^{\hat{\sigma}^{2}} \chi^{\prime}=\chi^{\prime}$ for all unipotent characters $\chi^{\prime} \in \operatorname{Irr}\left(\mathbf{L}^{F}\right)$. Hence $\mathcal{O} \mathbf{L}^{F} c \cong \mathcal{O} \mathbf{L}^{F} \hat{\sigma}^{2}(c)$.

\section{Sporadic groups, exceptional COVERING Groups, AND ALTERNATING GROUPS}

We give further analogues of results of [19] over $\mathcal{O}$. Let $b$ be a block of $\mathcal{O} G$ for a finite group $G$.

Proposition 7.1. Suppose that $G$ is a covering group of the sporadic simple group or the Tits group. Then $\mathcal{O} G \hat{\sigma}(b) \cong \mathcal{O} G b$.

Proof. If $b$ has cyclic defect, we apply Proposition 2.6. For the remaining cases, the checks done in [19] in GAP [21] show that $\hat{\sigma}(b)=b$.

Next we treat the exceptional covering groups of simple groups. The list in [19] was incomplete, so here we include a full proof over $\mathcal{O}$ for the complete list.

Proposition 7.2. Let $G$ be one of the following exceptional covering groups:

2. $L_{2}(4), 2 . L_{3}(2), 2 . L_{3}(4), 4_{1} . L_{3}(4), 4_{2} . L_{3}(4), 6 . L_{3}(4), 4^{2} . L_{3}(4)$, $12_{1} . L_{3}(4), 12_{2} . L_{3}(4),\left(4^{2} \times 3\right) . L_{3}(4), 2 . L_{4}(2), 2 . U_{4}(2), 2 . U_{6}(2)$, $6 . U_{6}(2), 2^{2} . U_{6}(2),\left(2^{2} \times 3\right) . U_{6}(2), 3 . A_{6}, 6 . A_{6}, 2 . S_{6}(2), 2 . S z(8)$, $2^{2} . S z(8), 2 . O_{8}^{+}(2), 2^{2} . O_{8}^{+}(2), 2 . G_{2}(4), 2 . F_{4}(2), 2 .{ }^{2} E_{6}(2), 6 .{ }^{2} E_{6}(2)$, $2^{2} .{ }^{2} E_{6}(2),\left(2^{2} \times 3\right) .{ }^{2} E_{6}(2), 3_{1} . U_{4}(3), 3_{2} . U_{4}(3), 6_{1} . U_{4}(3), 6_{2} . U_{4}(3)$, $3^{2} . U_{4}(3), 12_{1} . U_{4}(3), 12_{2} . U_{4}(3),\left(3^{2} \times 4\right) . U_{4}(3), 3 . O_{7}(3), 6 . O_{7}(3)$, 3. $G_{2}(3), 3 . A_{7}, 6 . A_{7}$.

Then $\mathcal{O} G b \cong \mathcal{O} G \hat{\sigma}(b)$.

Proof. We may assume that $b$ has noncyclic defect groups. By Lemma 2.10 we may also assume that $Z(G)_{\ell^{\prime}}$ is cyclic.

We see from checking in GAP [21] that in most cases the collections of $\ell$-blocks of $G$ with equal, noncyclic defect and the same number and degrees of characters, none of which are rational valued, have size 1 . Where there exists such a collection of size greater than 1 , it has size 2 . Suppose that $b_{1}$ and $b_{2}$ are nonprincipal $\ell$-blocks of $G$ with equal, noncyclic defect and the same number and degrees of characters, none of which are rational valued. Then in all but two situations, it is possible to check directly in GAP 21] that there exists a single block $\hat{b}$ of a finite group $\hat{G}$ with $G \unlhd \hat{G}$ such that $\hat{b}$ covers both $b_{1}$ and $b_{2}$. Hence $\mathcal{O} G b_{1} \cong \mathcal{O} G b_{2}$ via conjugation by an element of $\hat{G}$.

Finally, suppose that we are in one of the two remaining cases - that is, either $\ell=2$ and $G=\left(4^{2} \times 3\right) \cdot L_{3}(4)$, or $\ell=3$ and $G=\left(3^{2} \times 4\right) \cdot U_{4}(3)$. Suppose that $b$ is a nonprincipal $\ell$-block of $G$ with noncyclic defect groups which contains no rational valued character, and let $\bar{b}$ be the unique block of $G / Z(G)_{\ell}$ dominated by $b$. Then if $\ell=2$ and $G=\left(4^{2} \times 3\right) \cdot L_{3}(4), \bar{b}$ is one of two 2-blocks of $3 . L_{3}(4)$ which are faithful 
on the Sylow 3-subgroup of $G / Z(G)$. If $\ell=3$ and $G=\left(3^{2} \times 4\right) \cdot U_{4}(3)$, then $\bar{b}$ is one of two 3-blocks of $4 . U_{4}(3)$ which are faithful on the Sylow 2-subgroup of $G / Z(G)$. In both cases, by a GAP check there exists a single block $\hat{b}$ of a finite group $\hat{G}$ with $G / Z(G)_{\ell} \unlhd \hat{G}$ covering both the relevant blocks of $G / Z(G)_{\ell}$. In other words, there exists an automorphism of $G / Z(G)_{\ell}$ sending $\bar{b}$ to $\hat{\sigma}(\bar{b})$. Further, in both cases, $G$ is a universal covering group of $G / Z(G)_{\ell}$. Since every automorphism of a quasisimple finite group lifts to an automorphism of its universal covering group (see Section 5.1 of [24]), it follows from Lemma 2.10] that $\mathcal{O} G b \cong \mathcal{O} G \hat{\sigma}(b)$.

Proposition 7.3. Let $G$ be a quasi-simple finite group such that $G / Z(G)$ is an alternating group. Then $\mathcal{O} G b \cong \mathcal{O} G \hat{\sigma}(b)$.

Proof. Let $\hat{G}$ denote a finite group such that $G \triangleleft \hat{G}$ and $\hat{G} / Z(\hat{G})$ is a symmetric group, and let $\hat{b}$ denote a block of $\mathcal{O} \hat{G}$ covering $b$. Since we may assume that $b$ and hence $\hat{b}$ does not have cyclic defect groups, by Lemma 2.10(i) and the proof of [19, Theorem 3.1], $\hat{\sigma}(\hat{b})=\hat{b}$. It follows that $b$ and $\hat{\sigma}(b)$ are both covered by $\hat{b}$, and therefore $\mathcal{O} G b \cong \mathcal{O} G \hat{\sigma}(b)$ via conjugation by an element of $\hat{G}$.

\section{Proof of the MAIN THEOREMS}

Theorem 1.1 is part of the following result.

Theorem 8.1. Let $G$ be a quasi-simple finite group, and let $\bar{G}=G / Z(G)$. Let $b$ be a block of $\mathcal{O} G$, and let $D$ be a defect group of $\mathcal{O} G b$. There exists a finite group $N$ and a block $c$ of $\mathcal{O} N$ such that $\mathcal{O} G b$ is Morita equivalent to $\mathcal{O} N c$ and $\mathcal{O} N c \cong \mathcal{O} N \hat{\sigma}^{r}(c)$ for some $r \leq 4$. Moreover,

(i) if $\bar{G}$ is not a finite group of Lie type of type $E_{8}$ in characteristic $p \neq \ell$, then $r \leq 2$, and

(ii) if $\bar{G}$ is a sporadic group, an alternating group, a finite group of Lie type in characteristic $\ell$, or a finite group of Lie type of type $A, B$, or $C$ in characteristic $p \neq \ell$, then $r=1$.

Consequently, $m f(k G \pi(b)) \leq m f(\mathcal{O} G b) \leq 4$ and $s f(\mathcal{O} G b) \leq 4|D|^{2}$ !. Unless $\bar{G}$ is a finite group of Lie type of type $E_{8}$ in characteristic $p \neq \ell, m f(\mathcal{O} G b) \leq 2$. If $\bar{G}$ is one of the groups in (ii), then $m f(\mathcal{O} G b)=1$.

Proof. We first consider the claim that there exist a finite group $N$ and a block $c$ of $\mathcal{O} N$ such that $\mathcal{O} G b$ is Morita equivalent to $\mathcal{O} N c$ and $\mathcal{O} N c \cong \mathcal{O} N \hat{\sigma}^{r}(c)$ with bounds for $r$ as in the statement. For $G$ an exceptional covering group or $\bar{G}$ an alternating group, a sporadic group, the Tits group, or a finite group of Lie type in characteristic $\ell$ (including the Suzuki and Ree groups), then the result holds with $N=G$ and $c=b$ by Propositions 7.2, 7.1, 7.3, and 6.1, respectively. Proposition 6.2 proves the result for $\bar{G}$ a Suzuki or Ree group in characteristic $p \neq \ell$. Finally, suppose that $G$ is a nonexceptional cover of a finite group of Lie type in characteristic $p \neq \ell$. Then $G=\mathbf{G}^{F} / Z$ for $\mathbf{G}$ a simple simply connected algebraic group over $\overline{\mathbb{F}}_{p}, F: \mathbf{G} \rightarrow \mathbf{G}$ a Frobenius morphism, and $Z$ a central subgroup of $\mathbf{G}^{F}$. Hence the result holds by Propositions 5.1, 5.2, 5.3, 5.4, 5.5, 5.6, and 5.9, Note that although we assume in Section 5 that $Z$ is an $\ell$-group, this is enough to show that the results hold in general by Lemma 2.10, This proves the first part of the theorem.

Now $f(\mathcal{O} N c) \leq r$. Hence,

$$
m f(k G \pi(b)) \leq m f(\mathcal{O} G b)=m f(\mathcal{O} N c) \leq f(\mathcal{O} N c) \leq r,
$$


where the first and second inequalities hold by Proposition 2.5)(i), and the equality holds by Proposition 2.5(ii) and the fact that $\mathcal{O} G b$ and $\mathcal{O} N c$ are Morita equivalent. This proves the assertion about Morita Frobenius numbers.

Finally, since Morita equivalence between blocks of finite group algebras preserves orders of defect groups, the defect groups of $\mathcal{O} N c$ have order $|D|$. Hence

$$
s f(\mathcal{O} G b)=s f(\mathcal{O} N c) \leq f(\mathcal{O} N c)|D|^{2} ! \leq r|D|^{2} ! \leq 4|D|^{2} !,
$$

where the equality holds by Proposition 2.5(iii) and the first inequality holds by Proposition 2.5(i).

Proof of Theorem 1.4. The result follows from the first part of Theorem 1.1, 27, Theorem 8.6], and [28, Theorem 1.4].

Proof of Theorem 1.6. This follows from the first part of Theorem 1.1 and Lemma 2.8

\section{ACKNOWLEDGMents}

The authors thank Marc Cabanes and François Digne for their help in clearing up a point in the proof of Lemma 3.1. The authors also thank Gunter Malle and the referee for their careful reading and corrections.

It is a pleasure to thank the Mathematical Sciences Research Institute for its hospitality.

\section{REFERENCES}

[1] J. L. Alperin, Local representation theory, The Santa Cruz Conference on Finite Groups (Univ. California, Santa Cruz, Calif., 1979), Proc. Sympos. Pure Math., vol. 37, Amer. Math. Soc., Providence, R.I., 1980, pp. 369-375. MR604606

[2] David Benson and Radha Kessar, Blocks inequivalent to their Frobenius twists, J. Algebra 315 (2007), no. 2, 588-599, DOI 10.1016/j.jalgebra.2007.03.044. MR2351880

[3] Cédric Bonnafé, Quasi-isolated elements in reductive groups, Comm. Algebra 33 (2005), no. 7, 2315-2337, DOI 10.1081/AGB-200063602. MR2153225

[4] Cédric Bonnafé, Sur les caractères des groupes réductifs finis à centre non connexe: applications aux groupes spéciaux linéaires et unitaires (French, with English and French summaries), Astérisque 306 (2006), vi+165. MR2274998

[5] Cédric Bonnafé, Jean-François Dat, and Raphaël Rouquier, Derived categories and DeligneLusztig varieties II, Ann. of Math. (2) 185 (2017), no. 2, 609-670, DOI 10.4007/annals.2017.185.2.5. MR3612005

[6] Michel Broué, Gunter Malle, and Jean Michel, Generic blocks of finite reductive groups: Représentations unipotentes génériques et blocs des groupes réductifs finis, Astérisque 212 (1993), 7-92. MR 1235832

[7] Marc Cabanes, Local methods for blocks of finite simple groups, Local representation theory and simple groups, EMS Ser. Lect. Math., Eur. Math. Soc., Zürich, 2018, pp. 179-265. MR.3821140

[8] Marc Cabanes and Michel Enguehard, On unipotent blocks and their ordinary characters, Invent. Math. 117 (1994), no. 1, 149-164, DOI 10.1007/BF01232237. MR.1269428

[9] Marc Cabanes and Michel Enguehard, On blocks of finite reductive groups and twisted induction, Adv. Math. 145 (1999), no. 2, 189-229, DOI 10.1006/aima.1998.1814. MR1704575

[10] Marc Cabanes and Michel Enguehard, Representation theory of finite reductive groups, New Mathematical Monographs, vol. 1, Cambridge University Press, Cambridge, 2004. MR2057756

[11] Joseph Chuang and Radha Kessar, On perverse equivalences and rationality, European Congress of Mathematics, Eur. Math. Soc., Zürich, 2018, pp. 257-262. MR3887770

[12] P. Deligne and G. Lusztig, Representations of reductive groups over finite fields, Ann. of Math. (2) 103 (1976), no. 1, 103-161, DOI 10.2307/1971021. MR0393266 
[13] François Digne and Jean Michel, Representations of finite groups of Lie type, London Mathematical Society Student Texts, vol. 21, Cambridge University Press, Cambridge, 1991. MR.1118841

[14] C. W. Eaton, F. Eisele, and M. Livesey, Donovan's conjecture, blocks with abelian defect groups and discrete valuation rings, Mathematische Zeitschrift, 2019.

[15] Charles W. Eaton, Radha Kessar, Burkhard Külshammer, and Benjamin Sambale, 2-blocks with abelian defect groups, Adv. Math. 254 (2014), 706-735, DOI 10.1016/j.aim.2013.12.024. MR3161112

[16] Charles W. Eaton and Michael Livesey, Donovan's conjecture and blocks with abelian defect groups, Proc. Amer. Math. Soc. 147 (2019), no. 3, 963-970, DOI 10.1090/proc/14316. MR.3896046

[17] C. W. Eaton and M. Livesey, Towards Donovan's conjecture for abelian defect groups, J. Algebra 519, (2019), 39-61.

[18] Michel Enguehard, Sur les l-blocs unipotents des groupes réductifs finis quand l est mauvais (French), J. Algebra 230 (2000), no. 2, 334-377, DOI 10.1006/jabr.2000.8318. MR.1775796

[19] Niamh Farrell, On the Morita Frobenius numbers of blocks of finite reductive groups, J. Algebra 471 (2017), 299-318, DOI 10.1016/j.jalgebra.2016.08.043. MR3569187

[20] Farrell, N. Rationality of blocks of quasi-simple finite groups. PhD thesis, City, University of London, 2017.

[21] The GAP Group, GAP - Groups, Algorithms, and Programming, Version 4.7.9, 2015.

[22] Meinolf Geck, Character values, Schur indices and character sheaves, Represent. Theory 7 (2003), 19-55, DOI 10.1090/S1088-4165-03-00170-5. MR1973366

[23] Meinolf Geck, Gerhard Hiss, Frank Lübeck, Gunter Malle, and Götz Pfeiffer, CHEVIE-a system for computing and processing generic character tables: Computational methods in Lie theory (Essen, 1994), Appl. Algebra Engrg. Comm. Comput. 7 (1996), no. 3, 175-210, DOI 10.1007/BF01190329. MR 1486215

[24] Daniel Gorenstein, Richard Lyons, and Ronald Solomon, The classification of the finite simple groups. Number 4. Part II. Chapters 1-4, Mathematical Surveys and Monographs, vol. 40, American Mathematical Society, Providence, RI, 1999. Uniqueness theorems; With errata: The classification of the finite simple groups. Number 3. Part I. Chapter A [Amer. Math. Soc., Providence, RI, 1998; MR1490581 (98j:20011)]. MR.1675976

[25] G. Hiss, Zerlegungszahlen endlicher Gruppen vom Lie-Typ in nicht-definierender Charakteristik, Habilitationsschrift, 1990.

[26] Gerhard Hiss, On a question of Brauer in modular representation theory of finite groups, Representation theory of finite groups and related topics (Japanese) (Kyoto, 1998), Sūrikaisekikenkyūsho Kōkyūroku 1149 (2000), 21-29. MR,1796360

[27] Gerhard Hiss and Radha Kessar, Scopes reduction and Morita equivalence classes of blocks in finite classical groups. II, J. Algebra 283 (2005), no. 2, 522-563, DOI 10.1016/j.jalgebra.2004.08.030. MR 2111208

[28] Radha Kessar, A remark on Donovan's conjecture, Arch. Math. (Basel) 82 (2004), no. 5, 391-394, DOI 10.1007/s00013-004-4880-8. MR2061445

[29] Radha Kessar and Markus Linckelmann, Descent of equivalences and character bijections, Geometric and topological aspects of the representation theory of finite groups, Springer Proc. Math. Stat., vol. 242, Springer, Cham, 2018, pp. 181-212. MR3901160

[30] Radha Kessar and Gunter Malle, Quasi-isolated blocks and Brauer's height zero conjecture, Ann. of Math. (2) 178 (2013), no. 1, 321-384, DOI 10.4007/annals.2013.178.1.6. MR3043583

[31] Markus Linckelmann, The block theory of finite group algebras. Vol. II, London Mathematical Society Student Texts, vol. 92, Cambridge University Press, Cambridge, 2018. MR 3821517

[32] M. Linckelmann, The strong Frobenius numbers for cyclic defect blocks are equal to one, Comm. Algebra (2019), 1-8.

[33] George Lusztig, Characters of reductive groups over a finite field, Annals of Mathematics Studies, vol. 107, Princeton University Press, Princeton, NJ, 1984. MR742472

[34] G. Lusztig, On the representations of reductive groups with disconnected centre: Orbites unipotentes et représentations, I, Astérisque 168 (1988), 10, 157-166. MR.1021495

[35] Gunter Malle and Donna Testerman, Linear algebraic groups and finite groups of Lie type, Cambridge Studies in Advanced Mathematics, vol. 133, Cambridge University Press, Cambridge, 2011. MR2850737 
[36] Hirosi Nagao and Yukio Tsushima, Representations of finite groups, Academic Press, Inc., Boston, MA, 1989. Translated from the Japanese. MR.998775

[37] Jeremy Rickard, Splendid equivalences: derived categories and permutation modules, Proc. London Math. Soc. (3) 72 (1996), no. 2, 331-358, DOI 10.1112/plms/s3-72.2.331. MR1367082

[38] M. Schönert et al, GAP - Groups, Algorithms, and Programming - version 3 release 4 patchlevel 4, Lehrstuhl D für Mathematik, Rheinisch Westfälische Technische Hochschule, Aachen, Germany, 1997.

[39] Jean-Pierre Serre, Local fields, Graduate Texts in Mathematics, vol. 67, Springer-Verlag, New York-Berlin, 1979. Translated from the French by Marvin Jay Greenberg. MR554237

[40] Jay Taylor, Finding characters satisfying a maximal condition for their unipotent support, J. Pure Appl. Algebra 218 (2014), no. 3, 474-496, DOI 10.1016/j.jpaa.2013.06.016. MR3124212

Fachbereich Mathematik, TU Kaiserslautern, Postfach 3049, 67653 Kaiserslautern, GERMANY

Email address: farrell@mathematik.uni-kl.de

Department of Mathematics, City, University of London, Northampton Square, EC1V 0HB London, United Kingdom

Email address: radha.kessar.1@city.ac.uk 